\title{
Performance Analysis of Multi-Dimensional ESPRIT-Type Algorithms for Arbitrary and Strictly Non-Circular Sources with Spatial Smoothing
}

\author{
Jens Steinwandt*, Student Member, IEEE, Florian Roemer, Senior Member, IEEE, \\ Martin Haardt, Senior Member, IEEE, and Giovanni Del Galdo, Member, IEEE
}

\begin{abstract}
Spatial smoothing is a widely used preprocessing scheme to improve the performance of high-resolution parameter estimation algorithms in case of coherent signals or if only a small number of snapshots is available. In this paper, we present a first-order performance analysis of the spatially smoothed versions of $R$-D Standard ESPRIT and $R$-D Unitary ESPRIT for sources with arbitrary signal constellations as well as $R$ D NC Standard ESPRIT and $R$-D NC Unitary ESPRIT for strictly second-order (SO) non-circular (NC) sources. The derived expressions are asymptotic in the effective signal-to-noise ratio (SNR), i.e., the approximations become exact for either high SNRs or a large sample size. Moreover, no assumptions on the noise statistics are required apart from a zero-mean and finite SO moments. We show that both $R$-D NC ESPRIT-type algorithms with spatial smoothing perform asymptotically identical in the high effective SNR regime. Generally, the performance of spatial smoothing based algorithms depends on the number of subarrays, which is a design parameter and needs to be chosen beforehand. In order to gain more insights into the optimal choice of the number of subarrays, we simplify the derived analytical $R$-D mean square error (MSE) expressions for the special case of a single source. The obtained MSE expression explicitly depends on the number of subarrays in each dimension, which allows us to analytically find the optimal number of subarrays for spatial smoothing. Based on this result, we additionally derive the maximum asymptotic gain from spatial smoothing and explicitly compute the asymptotic efficiency for this special case. All the analytical results are verified by simulations.
\end{abstract}

Index Terms-Spatial smoothing, ESPRIT, non-circular sources, performance analysis, DOA estimation.

\section{INTRODUCTION}

$\mathbf{T}$ HE problem of high resolution parameter estimation from multi-dimensional ( $R$-D) signals with $R \geq 1$ has long been a fundamental research area in the field of array signal processing. Such a task, e.g., estimating the directions of arrival, directions of departures, frequencies, Doppler shifts, etc. arises in a wide range of applications including radar [2], sonar [3], channel sounding [4], [5], and wireless communications [6]. $R$-D ESPRIT-type parameter estimation

Minor parts of this paper have been published at the 2014 IEEE Int. Conf. on Acoustics, Speech, and Signal Proc. (ICASSP) [1].

The authors J. Steinwandt, F. Roemer, M. Haardt, and G. Del Galdo are with Ilmenau University of Technology, P.O. Box 100565, D98684 Ilmenau, Germany, e-mail: \{jens.steinwandt, florian.roemer, martin.haardt, giovanni.delgaldo\}@tu-ilmenau.de, phone: +49 (3677) 69-2613, web: http://www.tu-ilmenau.de/crl and http://www.tu-ilmenau.de/dvt.

F. Roemer and G. Del Galdo are also with the Fraunhofer Institute for Integrated Circuits IIS

* corresponding author algorithms [7] have attracted considerable attention due to their fully algebraic estimates and their low complexity. Hence, their analytical performance assessment has also been of great research interest. Two fundamental performance analysis concepts for 1-D parameter estimation have been established in [8] and [9]. While [8] relies on the eigenvector distribution of the sample covariance matrix and is only asymptotic in the sample size $N$, the framework in [9] provides an explicit firstorder approximation of the parameter estimation error based on the superposition of the signal component by a small noise perturbation. The latter is asymptotic in the effective signal-tonoise ratio (SNR), i.e., the results become accurate for either high SNRs or a large sample size. Therefore, [9] is more general than [8] as it is even valid for $N=1$ if the SNR is sufficiently high. In [10], [11], this performance analysis framework was extended to $R$-D parameter estimation, where no assumptions on the noise statistics apart from a zero mean and finite second-order ( $\mathrm{SO}$ ) moments are required for the analytical mean square error (MSE) expressions.

Many authors have shown that taking advantage of existing properties of the observed signals such as their strictly SO noncircular (NC) structure [12] helps to improve the performance of conventional parameter estimation algorithms. Examples of such NC signals include BPSK, PAM, and ASK-modulated signals. They are of practical relevance in wireless communications, cognitive radio, GNSS satellite systems etc., when strictly non-circular sources are known to be present, or in radar, tracking, channel sounding, etc., where the transmit signals can be designed as strictly non-circular. Recently, a number of improved subspace-based parameter estimation schemes, e.g., NC MUSIC [13]-[15], NC Root-MUSIC [16], NC Standard ESPRIT [17], and NC Unitary ESPRIT [18], [19] have been developed. It has been demonstrated that exploiting the prior knowledge on the signals' strict noncircularity significantly improves the estimation accuracy and doubles the number of identifiable sources [18]. The analytical performance of the MUSIC and ESPRIT-based NC algorithms has been investigated in [13], [19], [20]. For the special case of a single source, it was shown in [10] along with [19] that neither forward-backward averaging (FBA) nor NC preprocessing in combination with ESPRIT-type algorithms improve the asymptotic MSE. The more general case of coexisting circular and strictly non-circular signals has been considered in [21] $-[23]$.

The aforementioned $\mathrm{NC}$ and conventional methods are 
known to yield a high resolution even in the case of correlated sources. However, they fail when more than two signals 1 are coherent (fully correlated) or if $N=1$, as both render the signal covariance matrix rank-deficient. In practice, coherent signals often occur in a multipath environment [25] and the single snapshot case is often encountered in, e.g., channel sounding [5], co-prime arrays [26], tracking [27]. Assuming a uniform array geometry, preprocessing via spatial smoothing [28]-[30] can be applied to estimate the parameters of coherent signals. Spatial smoothing decorrelates coherent signals by averaging the data received by a number of subarrays $L$. As the resulting estimation error depends on $L$, this is a design parameter that can be optimized to achieve the best estimation accuracy. Several performance analyses of parameter estimation schemes using spatial smoothing based on the framework [8], which is, however, only asymptotic in $N$, have been presented in [31]-[37]. While [31]-[33] consider spatially smoothed MUSIC-type algorithms, the references [34]-[36] study ESPRIT-type algorithms. In [37], a performance analysis for an interpolated spatial smoothing algorithm for non-uniform linear arrays was proposed. The special case of spatial smoothing for a single source was considered in [32], [33], and in [34] for harmonic retrieval. It was observed that in this case a gain from spatial smoothing can be achieved. However, these existing performance analysis results only concern the 1-D parameter estimation. Analytical expressions for $R$-D parameter estimation algorithms such as $R$-D Standard ESPRIT and $R$-D Unitary ESPRIT with spatial smoothing as well as their recently proposed NC-versions $R$ D NC Standard ESPRIT and $R$-D NC Unitary ESPRIT with spatial smoothing have not been reported in the literature.

Therefore, in this paper, we present a first-order performance analysis for the spatially smoothed versions of $R$-D Standard ESPRIT and $R$-D Unitary ESPRIT as well as $R$-D NC Standard ESPRIT and $R$-D NC Unitary ESPRIT based on the more general framework in [9], which is asymptotic in the high effective SNR. We assume a uniform $R$-D array geometry and use least squares (LS) to solve the shift invariance equations. However, as LS and total least squares (TLS) have been shown to perform asymptotically identical [8], the results obtained for LS are also valid for TLS. The derived closedform MSE expressions are explicit in the noise realizations such that apart from a zero mean and finite SO moments, no further assumptions on the noise statistics are required. We show that due to the NC preprocessing both $R$-D NC ESPRITtype algorithms with spatial smoothing perform identical in the high effective SNR. Further insights into the dependence of the MSE expressions on the physical parameters are provided by the case study of a single source. For this case, we first show that $R$-D spatial smoothing improves the estimation accuracy and that all the considered spatial smoothing based $R$-D ESPRIT-type algorithms provide the same MSE result, i.e., asymptotically, no additional gain is obtained from FBA and NC preprocessing. Based on these results, we analytically find the optimal number of subarrays $L$ that minimizes the

\footnotetext{
${ }^{1}$ Two coherent signals can be separated by forward-backward averaging (FBA) if the array phase reference is not located at the array centroid [24].
}

MSE in each of the $R$ dimensions, which extends the 1-D results in [31]-[37]. This enables us to compute the maximum asymptotic $R$-D spatial smoothing gain for a single source in closed-form. Additionally, we analytically compute the asymptotic efficiency of the spatial smoothing based algorithms for $R=1$.

This paper is organized as follows: The $R$-D data model and the preprocessing for NC sources are introduced in Section III. Section IIII reviews $R$-D spatial smoothing for ESPRITtype and NC ESPRIT-type algorithms. Their performance analysis is presented in Section $\nabla$ before the special case of a single source is analyzed in Section VI Section VII illustrates the numerical results, and concluding remarks are drawn in Section VIII

Notation: We use lower-case bold-face letters for column vectors and upper-case bold-face letters for matrices. The superscripts ${ }^{\mathrm{T}},{ }^{*}, \mathrm{H},-1,+$ denote the transposition, complex conjugation, conjugate transposition, matrix inversion, and the Moore-Penrose pseudo inverse, respectively. The Kronecker product is denoted as $\otimes$ and the Khatri-Rao product (columnwise Kronecker product) as $\diamond$. The operator vec $\{\boldsymbol{A}\}$ stacks the columns of the matrix into a large column vector, $\operatorname{diag}\{\boldsymbol{a}\}$ returns a diagonal matrix with the elements of $\boldsymbol{a}$ placed on its diagonal, and blkdiag $\{\cdot\}$ creates a block diagonal matrix. The operator $\mathcal{O}\{\cdot\}$ denotes the highest order with respect to a parameter. The matrix $\Pi_{M}$ is the $M \times M$ exchange matrix with ones on its anti-diagonal and zeros elsewhere and $\underline{1}$ denotes the vector of ones. Moreover, $\operatorname{Re}\{\cdot\}$ and $\operatorname{Im}\{\cdot\}$ extract the real and imaginary part of a complex number and $\arg \{\cdot\}$ extracts its phase. Also, $\|\boldsymbol{x}\|_{2}$ represents the 2-norm of the vector $\boldsymbol{x}$, and $\mathbb{E}\{\cdot\}$ stands for the statistical expectation. Furthermore, we use the short hand notation

$$
\sum_{\underline{\ell}=\underline{\mathbf{1}}}^{\underline{\boldsymbol{L}}} x_{\underline{\underline{\ell}}}=\sum_{\ell_{1}=1}^{L_{1}} \sum_{\ell_{2}=1}^{L_{2}} \cdots \sum_{\ell_{R}=1}^{L_{R}} x_{\ell_{1}, \ldots, \ell_{R}}
$$

where $\underline{\boldsymbol{\ell}}=\left[\ell_{1}, \ldots, \ell_{r}, \ldots, \ell_{R}\right]$ and $\underline{\boldsymbol{L}}=\left[L_{1}, \ldots, L_{r}, \ldots, L_{R}\right]$ with $\ell_{r}=1, \ldots, L_{r}, r=1, \ldots, R$.

\section{Data Model}

In this section, we introduce the $R$-D data model for arbitrary signals followed by the NC data model for strictly non-circular signals.

\section{A. Data Model for Arbitrary Signals}

Suppose the measurement data is represented by $N$ subsequent observations of a noise-corrupted superposition of $d$ undamped exponentials sampled on a separable uniform $R$-D grid of size $M_{1} \times \ldots \times M_{R}$ [7]. The $t_{n}$-th time snapshot of the $R$-D measurements can be modeled as

$$
x_{m_{1}, \ldots, m_{R}}\left(t_{n}\right)=\sum_{i=1}^{d} s_{i}\left(t_{n}\right) \prod_{r=1}^{R} \mathrm{e}^{\mathrm{j}\left(m_{r}-1\right) \mu_{i}^{(r)}}+n_{m_{1}, \ldots, m_{R}}\left(t_{n}\right),
$$

where $m_{r}=1, \ldots, M_{r}, n=1, \ldots, N$, and $s_{i}\left(t_{n}\right)$ represents the complex amplitude of the $i$-th undamped exponential at the time instant $t_{n}$. Furthermore, $\mu_{i}^{(r)}$ is the $i$-th spatial frequency in the $r$-th mode, $i=1, \ldots, d, r=1, \ldots, R$, and 
$n_{m_{1}, \ldots, m_{R}}\left(t_{n}\right)$ denotes the zero-mean additive noise component. In the context of array signal processing, each of the $R$-D exponentials represents a narrow-band planar wavefront from a stationary far-field source and the complex amplitudes $s_{i}\left(t_{n}\right)$ describe the source symbols. The goal is to estimate the $R \cdot d$ spatial frequencies $\boldsymbol{\mu}_{i}=\left[\mu_{i}^{(1)}, \ldots, \mu_{i}^{(R)}\right]^{\mathrm{T}}, \forall i$, from (2). We assume that $d$ is known or has been estimated beforehand.

In order to obtain a more compact formulation of (2), we form the measurement matrix $\boldsymbol{X} \in \mathbb{C}^{M \times N}$ with $M=$ $\prod_{r=1}^{R} M_{r}$ by stacking the $R$ spatial dimensions and aligning the $N$ time snapshots as the columns. This way, $\boldsymbol{X}$ can be modeled as

$$
\boldsymbol{X}=\boldsymbol{A} \boldsymbol{S}+\boldsymbol{N} \in \mathbb{C}^{M \times N},
$$

where $S \in \mathbb{C}^{d \times N}$ represents the source symbol matrix, $\boldsymbol{N} \in \mathbb{C}^{M \times N}$ contains the noise samples, and $\boldsymbol{A}=$ $\left[\boldsymbol{a}\left(\boldsymbol{\mu}_{1}\right), \ldots, \boldsymbol{a}\left(\boldsymbol{\mu}_{d}\right)\right] \in \mathbb{C}^{M \times d}$ is the array steering matrix. The latter consists of the array steering vectors $\boldsymbol{a}\left(\boldsymbol{\mu}_{i}\right)$ corresponding to the $i$-th spatial frequency, which are given by

$$
\boldsymbol{a}\left(\boldsymbol{\mu}_{i}\right)=\boldsymbol{a}^{(1)}\left(\mu_{i}^{(1)}\right) \otimes \cdots \otimes \boldsymbol{a}^{(R)}\left(\mu_{i}^{(R)}\right) \in \mathbb{C}^{M \times 1},
$$

where $\boldsymbol{a}^{(r)}\left(\mu_{i}^{(r)}\right) \in \mathbb{C}^{M_{r} \times 1}$ is the array steering vector in the $r$-th mode. Alternatively, $\boldsymbol{A}$ can be expressed as

$$
\boldsymbol{A}=\boldsymbol{A}^{(1)} \diamond \boldsymbol{A}^{(2)} \diamond \cdots \diamond \boldsymbol{A}^{(R)},
$$

where $\boldsymbol{A}^{(r)}=\left[\boldsymbol{a}^{(r)}\left(\mu_{1}^{(r)}\right), \ldots, \boldsymbol{a}^{(r)}\left(\mu_{d}^{(r)}\right)\right] \in \mathbb{C}^{M_{r} \times d}$ represents the array steering matrix in the $r$-th mode. For an arbitrary phase reference along the $r$-th mode, $\boldsymbol{A}^{(r)}$ can be decomposed as [38] $\boldsymbol{A}^{(r)}=\overline{\boldsymbol{A}}^{(r)} \boldsymbol{\Delta}^{(r)}$, where $\overline{\boldsymbol{A}}^{(r)}=$ $\left[\overline{\boldsymbol{a}}^{(r)}\left(\mu_{1}^{(r)}\right), \cdots, \overline{\boldsymbol{a}}^{(r)}\left(\mu_{d}^{(r)}\right)\right] \in \mathbb{C}^{M_{r} \times d}$ satisfies $\overline{\boldsymbol{A}}^{(r)}=$ $\boldsymbol{\Pi}_{M_{r}} \overline{\boldsymbol{A}}^{(r)^{*}}$ and contains the steering vectors $\overline{\boldsymbol{a}}^{(r)}\left(\mu_{i}^{(r)}\right), i=$ $1, \ldots, d$, whose phase reference is located at the centroid of the $r$-th mode, i.e.,

$$
\overline{\boldsymbol{a}}^{(r)}\left(\mu_{i}^{(r)}\right)=\left[\begin{array}{lll}
\mathrm{e}^{-\mathrm{j} \frac{\left(M_{r}-1\right)}{2} \mu_{i}^{(r)}} & \cdots & \mathrm{e}^{\mathrm{j} \frac{\left(M_{r}-1\right)}{2} \mu_{i}^{(r)}}
\end{array}\right] .
$$

Furthermore, the diagonal matrix $\boldsymbol{\Delta}^{(r)}=\operatorname{diag}\left\{\mathrm{e}^{\mathrm{j} \delta^{(r)} \mu_{i}^{(r)}}\right\}_{i=1}^{d}$ defines the shifts of the phase reference $\delta^{(r)} \in$ $\left[\frac{-\left(M_{r}-1\right)}{2}, \frac{\left(M_{r}-1\right)}{2}\right]$ for each $\mu_{i}^{(r)}$. If the actual phase reference is at the array centroid of the $r$-th mode, we have $\delta^{(r)}=0, \boldsymbol{\Delta}^{(r)}=\boldsymbol{I}_{d}$, and consequently $\boldsymbol{A}^{(r)}=\overline{\boldsymbol{A}}^{(r)}$. Thus, we can rewrite $\boldsymbol{A}$ in (5) as [38] $\boldsymbol{A}=\overline{\boldsymbol{A}} \boldsymbol{\Delta}$, where $\overline{\boldsymbol{A}}=\overline{\boldsymbol{A}}^{(1)} \diamond \overline{\boldsymbol{A}}^{(2)} \diamond \cdots \diamond \overline{\boldsymbol{A}}^{(R)} \in \mathbb{C}^{M \times d}$ and $\boldsymbol{\Delta}=\boldsymbol{\Delta}^{(1)} \cdot \boldsymbol{\Delta}^{(2)} \cdot \ldots \cdot \boldsymbol{\Delta}^{(R)} \in \mathbb{C}^{d \times d}$. Again, if $\delta^{(r)}=0 \forall r$, we have $\boldsymbol{A}=\overline{\boldsymbol{A}}$. Using these relations, we obtain the model

$$
\boldsymbol{X}=\overline{\boldsymbol{A}} \boldsymbol{\Delta} \boldsymbol{S}+\boldsymbol{N}=\overline{\boldsymbol{A}} \overline{\boldsymbol{S}}+\boldsymbol{N} \in \mathbb{C}^{M \times N} .
$$

Due to the assumption that the $R$-D sampling grid is uniform, the array steering matrix $\boldsymbol{A}$ satisfies the shift invariance equations given by

$$
\tilde{\boldsymbol{J}}_{1}^{(r)} \overline{\boldsymbol{A}} \boldsymbol{\Phi}^{(r)}=\tilde{\boldsymbol{J}}_{2}^{(r)} \overline{\boldsymbol{A}}, \quad r=1, \ldots, R,
$$

where $\tilde{\boldsymbol{J}}_{1}^{(r)}$ and $\tilde{\boldsymbol{J}}_{2}^{(r)} \in \mathbb{R}^{\frac{M}{M_{r}}\left(M_{r}-1\right) \times M}$ are the effective $R$ D selection matrices, which select $M_{r}-1$ elements (maximum overlap) for the first and the second subarray in the $r$-th mode, respectively. They are compactly defined as
$\tilde{\boldsymbol{J}}_{k}^{(r)}=\boldsymbol{I}_{\prod_{l=1}^{r-1} M_{l}} \otimes \boldsymbol{J}_{k}^{(r)} \otimes \boldsymbol{I}_{\prod_{l=r+1}^{R} M_{l}}$ for $k=1,2$, where $\boldsymbol{J}_{k}^{(r)} \in \mathbb{R}^{\left(M_{r}-1\right) \times M_{r}}$ are the $r$-mode selection matrices for the first and second subarray [7]. The diagonal matrix $\boldsymbol{\Phi}^{(r)}=\operatorname{diag}\left\{\left[\mathrm{e}^{\mathrm{j} \mu_{1}^{(r)}}, \ldots, \mathrm{e}^{\mathrm{j} \mu_{d}^{(r)}}\right]\right\} \in \mathbb{C}^{d \times d}$ contains the spatial frequencies in the $r$-th mode to be estimated.

\section{B. Preprocessing for Strictly Non-Circular Signals}

A zero-mean complex random variable $Z=X+\mathrm{j} Y$ is said to be $\mathrm{SO}$ non-circular if $\mathbb{E}\left\{Z^{2}\right\} \neq 0$ holds, which implies that its real and its imaginary part are correlated. The degree of non-circularity is usually defined by the non-circularity coefficient [12]

$$
\kappa=\frac{\mathbb{E}\left\{Z^{2}\right\}}{\mathbb{E}\left\{|Z|^{2}\right\}}=|\kappa| \mathrm{e}^{\mathrm{j} \psi}, \quad 0 \leq|\kappa| \leq 1 .
$$

Random variables that satisfy $|\kappa|=0$ or $0<|\kappa|<1$ are called circularly symmetric or weak-sense SO non-circular, respectively. The case $|\kappa|=1$ describes a strictly SO noncircular (also referred to as rectilinear) random variable. The latter, which is considered in this work, implies a linear dependence between the real and the imaginary part of $Z$. Thus, $Z$ can be represented as a real-valued random variable $W$ which is rotated by a deterministic complex phase term $\mathrm{e}^{\mathrm{j} \varphi}$, i.e., $Z=W \mathrm{e}^{\mathrm{j} \varphi}$.

In a communication system, the case of strictly SO nocircular signals presumes that the sources transmit real-valued constellations (BPSK, ASK, Offset-QPSK after a derotation, etc.) whose symbol amplitudes in the complex plane at the receiver lie on lines with different phase rotations as the sources may have different transmission delays. Therefore, the symbol matrix $S$ in (3) can be decomposed as [18 $\boldsymbol{S}=\boldsymbol{\Psi} \boldsymbol{S}_{0}$, where $\boldsymbol{S}_{0} \in \mathbb{R}^{d \times N}$ is a real-valued symbol matrix and $\boldsymbol{\Psi}=\operatorname{diag}\left\{\mathrm{e}^{\mathrm{j} \varphi_{i}}\right\}_{i=1}^{d}$ contains stationary complex phase shifts on its diagonal that can be different for each source. Then, $\overline{\boldsymbol{S}}$ in (7) is given by $\overline{\boldsymbol{S}}=\Delta \boldsymbol{\Psi} \boldsymbol{S}_{0}=\boldsymbol{\Xi} \boldsymbol{S}_{0}$, where we have defined $\boldsymbol{\Xi}=\boldsymbol{\Delta} \boldsymbol{\Psi}=\operatorname{diag}\left\{\mathrm{e}^{\mathrm{j}\left(\varphi_{i}+\delta_{i}\right)}\right\}_{i=1}^{d}$ with $\delta_{i}=\sum_{r=1}^{R} \delta^{(r)} \mu_{i}^{(r)}$.

In order to take advantage of the strict non-circularity of the signals, we apply a preprocessing scheme to $(3)$ and define the augmented measurement matrix $\boldsymbol{X}^{(\mathrm{nc})} \in \mathbb{C}^{2 M \times N}$ as [18]

$$
\begin{aligned}
\boldsymbol{X}^{(\mathrm{nc})} & =\left[\begin{array}{c}
\boldsymbol{X} \\
\boldsymbol{\Pi}_{M} \boldsymbol{X}^{*}
\end{array}\right]=\left[\begin{array}{c}
\overline{\boldsymbol{A}} \\
\overline{\boldsymbol{A}} \boldsymbol{\Xi}^{*} \boldsymbol{\Xi}^{*}
\end{array}\right] \overline{\boldsymbol{S}}+\left[\begin{array}{c}
\boldsymbol{N} \\
\boldsymbol{\Pi}_{M} \boldsymbol{N}^{*}
\end{array}\right] \\
& =\overline{\boldsymbol{A}}^{(\mathrm{nc})} \overline{\boldsymbol{S}}+\boldsymbol{N}^{(\mathrm{nc})}
\end{aligned}
$$

where $\Pi_{M}$ is the $M \times M$ exchange matrix with ones on its antidiagonal and zeros elsewhere and we have used the property $\Pi_{M} \overline{\boldsymbol{A}}^{*}=\overline{\boldsymbol{A}}$. Moreover, $\overline{\boldsymbol{A}}^{(\mathrm{nc})} \in \mathbb{C}^{2 M \times d}$ and $N^{(\text {nc) }} \in \mathbb{C}^{2 M \times N}$ are the augmented array steering matrix and the augmented noise matrix, respectively.

It was shown in [19] that if the array steering matrix $\bar{A}$ is shift-invariant (8), then $\bar{A}^{(n c)}$ is also shift-invariant and satisfies

$$
\tilde{\boldsymbol{J}}_{1}^{(\mathrm{nc})(r)} \overline{\boldsymbol{A}}^{(\mathrm{nc})} \boldsymbol{\Phi}^{(r)}=\tilde{\boldsymbol{J}}_{2}^{(\mathrm{nc})(r)} \overline{\boldsymbol{A}}^{(\mathrm{nc})}, \quad r=1, \ldots, R,
$$

where $\tilde{\boldsymbol{J}}_{k}^{(\mathrm{nc})(r)}=\boldsymbol{I}_{\prod_{l=1}^{r-1} M_{l}} \otimes \boldsymbol{J}_{k}^{(\mathrm{nc})(r)} \otimes \boldsymbol{I}_{\prod_{l=r+1}^{R} M_{l}}$ and $\boldsymbol{J}_{k}^{(\mathrm{nc})(r)}=\boldsymbol{I}_{2} \otimes \boldsymbol{J}_{k}^{(r)}, k=1,2$. Note that the extended 
dimensions of $\overline{\boldsymbol{A}}^{(\mathrm{nc})}$ can be interpreted as a virtual doubling of the number of sensors, which leads to a lower estimation error and doubles the number of resolvable sources [18].

\section{III. $R$-D SPATIAL SMOOTHING}

In this section, we first apply $R$-D spatial smoothing to the data model for arbitrary signals in (3) before considering the strictly non-circular data model in (10).

\section{A. R-D Spatial Smoothing for Signals with Arbitrary Signal Constellations}

In the case of coherent signals (fully correlated), or for a single snapshot $N=1$, the symbol matrix $\bar{S}$ becomes row rank deficient, i.e., $\operatorname{rank}\{\overline{\boldsymbol{S}}\}<d$. If only two signals are coherent, forward-backward averaging (FBA) [30] can separate these signals if the corresponding diagonal elements of $\boldsymbol{\Delta}$ are distinct [24], i.e., the phase reference is not at the array centroid. For more than two coherent signals, however, the conventional subspace-based parameter estimators fail to estimate the directions of the coherent signals. In case of a uniform array geometry, spatial smoothing preprocessing can be applied to restore the full row rank $d$ of $\bar{S}$ albeit reducing the effective array aperture.

In order to perform $R$-D spatial smoothing, we apply 1-D spatial smoothing to each of the $R$ dimensions independently [7]. To this end, the $M_{r}$ uniform sampling grid points in the $r$-th dimension are divided into $L_{r}$ maximally overlapping subarrays, each containing $M_{\mathrm{sub}_{r}}=M_{r}-L_{r}+1$ elements. The corresponding $M_{\mathrm{sub}_{r}} \times M_{r}$ selection matrix for the $\ell_{r}$-th subarray, $1 \leq \ell_{r} \leq L_{r}$ for $1 \leq r \leq R$, is defined as

$$
\boldsymbol{J}_{\ell_{r}}^{\left(M_{r}\right)}=\left[\begin{array}{lll}
\mathbf{0}_{M_{\mathrm{sub}_{r}} \times\left(\ell_{r}-1\right)} & \boldsymbol{I}_{M_{\mathrm{sub}_{r}}} & \mathbf{0}_{M_{\mathrm{sub}_{r}} \times\left(L_{r}-\ell_{r}\right)}
\end{array}\right] .
$$

Next, we define the $L=\prod_{r=1}^{R} L_{r}$ multi-dimensional selection matrices

$$
\begin{aligned}
\boldsymbol{J}_{\underline{\ell}} & =\boldsymbol{J}_{\ell_{1}, \ldots, \ell_{R-1}, \ell_{R}} \\
& =\boldsymbol{J}_{\ell_{1}}^{\left(M_{1}\right)} \otimes \cdots \otimes \boldsymbol{J}_{\ell_{R-1}}^{\left(M_{R-1}\right)} \otimes \boldsymbol{J}_{\ell_{R}}^{\left(M_{R}\right)} \in \mathbb{R}^{M_{\mathrm{sub}} \times M}
\end{aligned}
$$

for $1 \leq \ell_{r} \leq L_{r}$ with $M_{\mathrm{sub}}=\prod_{r=1}^{R} M_{\mathrm{sub}_{r}}$. Then, the spatially smoothed data matrix $\boldsymbol{X}_{\mathrm{SS}} \in \mathbb{C}^{M_{\text {sub }} \times N L}$, which is subsequently processed instead of $\boldsymbol{X}$, is given by

$$
\begin{aligned}
\boldsymbol{X}_{\mathrm{SS}}= & {\left[\begin{array}{llll}
\boldsymbol{J}_{1, \cdots, 1,1} \boldsymbol{X} & \boldsymbol{J}_{1, \cdots, 1,2} \boldsymbol{X} & \cdots & \boldsymbol{J}_{1, \cdots, 1, L_{R}} \boldsymbol{X} \\
& \boldsymbol{J}_{1, \cdots, 2,1} \boldsymbol{X} & \cdots & \boldsymbol{J}_{L_{1}, \cdots, L_{R-1}, L_{R}} \boldsymbol{X}
\end{array}\right] } \\
= & {\left[\begin{array}{llll}
\boldsymbol{J}_{1, \cdots, 1,1} \overline{\boldsymbol{A}} \overline{\boldsymbol{S}} & \cdots & \boldsymbol{J}_{L_{1}, \cdots, L_{R-1}, L_{R}} \overline{\boldsymbol{A}} \overline{\boldsymbol{S}}
\end{array}\right] } \\
& +\left[\begin{array}{llll}
\boldsymbol{J}_{1, \cdots, 1,1} \boldsymbol{N} & \cdots & \boldsymbol{J}_{L_{1}, \cdots, L_{R-1}, L_{R}} \boldsymbol{N}
\end{array}\right]
\end{aligned}
$$

Note that by using (5) and (13), the array steering matrix of the $\ell$-th subarray in all $R$ modes can be expressed as

$$
\begin{aligned}
& \boldsymbol{J}_{\ell_{1}, \ldots, \ell_{R-1}, \ell_{R}} \overline{\boldsymbol{A}}=\left(\boldsymbol{J}_{\ell_{1}}^{\left(M_{1}\right)} \overline{\boldsymbol{A}}^{(1)}\right) \diamond \cdots \diamond\left(\boldsymbol{J}_{\ell_{R}}^{\left(M_{R}\right)} \overline{\boldsymbol{A}}^{(R)}\right) \\
& =\left(\overline{\boldsymbol{A}}_{1}^{(1)}\left(\boldsymbol{\Phi}^{(1)}\right)^{\ell_{1}-1}\right) \diamond \cdots \diamond\left(\overline{\boldsymbol{A}}_{1}^{(R)}\left(\boldsymbol{\Phi}^{(R)}\right)^{\ell_{R}-1}\right) \\
& =\overline{\boldsymbol{A}}_{\mathrm{SS}} \boldsymbol{\Phi}_{\ell_{1}, \ldots, \ell_{R-1}, \ell_{R}},
\end{aligned}
$$

where we have defined $\overline{\boldsymbol{A}}_{1}^{(r)}=\boldsymbol{J}_{1_{r}}^{\left(M_{r}\right)} \overline{\boldsymbol{A}}^{(r)} \in \mathbb{C}^{M_{\mathrm{sub}_{r}} \times d}$, $\overline{\boldsymbol{A}}_{\mathrm{SS}}=\overline{\boldsymbol{A}}_{1}^{(1)} \diamond \cdots \diamond \overline{\boldsymbol{A}}_{1}^{(R)}=\boldsymbol{J}_{1, \cdots, 1,1} \overline{\boldsymbol{A}} \in \mathbb{C}^{M_{\mathrm{sub}} \times d}$, and

$$
\boldsymbol{\Phi}_{\ell_{1}, \ldots, \ell_{R-1}, \ell_{R}}=\prod_{r=1}^{R}\left(\boldsymbol{\Phi}^{(r)}\right)^{\ell_{r}-1} .
$$

Consequently, we can rewrite (14) by applying (15) as

$$
\boldsymbol{X}_{\mathrm{SS}}=\overline{\boldsymbol{A}}_{\mathrm{SS}} \boldsymbol{\Phi}\left(\boldsymbol{I}_{L} \otimes \overline{\boldsymbol{S}}\right)+\boldsymbol{N}_{\mathrm{SS}}=\boldsymbol{X}_{\mathrm{SS}_{0}}+\boldsymbol{N}_{\mathrm{SS}}
$$

where $\boldsymbol{\Phi}=\left[\boldsymbol{\Phi}_{1, \ldots, 1,1}, \cdots, \boldsymbol{\Phi}_{1, \ldots, 1, L_{R}}, \boldsymbol{\Phi}_{1, \ldots, 2,1}, \cdots\right.$ $\left.\boldsymbol{\Phi}_{L_{1}, \ldots, L_{R-1}, L_{R}}\right] \in \mathbb{C}^{d \times L d}, \boldsymbol{X}_{\mathrm{SS}_{0}} \in \mathbb{C}^{M_{\mathrm{sub}} \times N L}$ is the noisefree spatially smoothed data matrix, and $\boldsymbol{N}_{\mathrm{SS}} \in \mathbb{C}^{M_{\mathrm{sub}} \times N L}$ is the spatially smoothed noise. Thus, spatial smoothing preprocessing reduces the array aperture to $M_{\text {sub }}$ sensors and increases the number of snapshots by the factor $L$.

It is apparent that $\overline{\boldsymbol{A}}_{\mathrm{SS}}$ still satisfies the shift-invariance equation and we can write

$$
\tilde{\boldsymbol{J}}_{\mathrm{SS}}^{(r)} \overline{\boldsymbol{A}}_{\mathrm{SS}} \boldsymbol{\Phi}^{(r)}=\tilde{\boldsymbol{J}}_{\mathrm{SS}_{2}}^{(r)} \overline{\boldsymbol{A}}_{\mathrm{SS}}, \quad r=1, \ldots, R,
$$

where $\tilde{\boldsymbol{J}}_{\mathrm{SS}_{1}}^{(r)}$ and $\tilde{\boldsymbol{J}}_{\mathrm{SS}_{2}}^{(r)} \in \mathbb{R}^{\frac{M_{\mathrm{sub}}}{M_{\mathrm{sub}_{r}}}\left(M_{\mathrm{sub}_{r}}-1\right) \times M_{\mathrm{sub}}}$ are the $R$ D selection matrices that select $M_{\mathrm{sub}_{r}}-1$ elements for the first and the second subarray in the $r$-th mode, respectively. They are compactly defined as $\tilde{\boldsymbol{J}}_{\mathrm{SS}_{k}}^{(r)}=\boldsymbol{I}_{\prod_{l=1}^{r-1} M_{\mathrm{sub}_{l}}} \otimes \boldsymbol{J}_{\mathrm{SS}_{k}}^{(r)} \otimes$ $\boldsymbol{I}_{\prod_{l=r+1}^{R} M_{\mathrm{sub}_{l}}}$ for $k=1,2$, where $\boldsymbol{J}_{\mathrm{SS}_{k}}^{(r)} \in \mathbb{R}^{\left(M_{\mathrm{sub}_{r}}-1\right) \times M_{\mathrm{sub}_{r}}}$ are the $r$-mode selection matrices for the first and second subarray. As 17 holds, the $R \cdot d$ spatial frequencies can be estimated by applying $R$-D ESPRIT-type algorithms to $\boldsymbol{X}_{\mathrm{SS}}$. In $R$-D Standard ESPRIT, the signal subspace $\hat{\boldsymbol{U}}_{\mathrm{SS}_{\mathrm{s}}} \in \mathbb{C}^{M_{\mathrm{sub}} \times d}$ is estimated by computing the $d$ dominant left singular vectors of $\boldsymbol{X}_{\mathrm{SS}}$. As $\overline{\boldsymbol{A}}_{\mathrm{SS}}$ and $\hat{\boldsymbol{U}}_{\mathrm{SS}_{\mathrm{S}}}$ span approximately the same column space, a non-singular matrix $\boldsymbol{T} \in \mathbb{C}^{d \times d}$ can be found such that $\overline{\boldsymbol{A}}_{\mathrm{SS}} \approx \hat{\boldsymbol{U}}_{\mathrm{SS}_{\mathrm{S}}} \boldsymbol{T}$. Using this relation, the overdetermined set of $R$ shift invariance equations (17) can be expressed in terms of the estimated signal subspace, yielding

$$
\tilde{\boldsymbol{J}}_{\mathrm{SS}_{1}}^{(r)} \hat{\boldsymbol{U}}_{\mathrm{SS}_{\mathrm{s}}} \boldsymbol{\Gamma}^{(r)} \approx \tilde{\boldsymbol{J}}_{\mathrm{SS}_{2}}^{(r)} \hat{\boldsymbol{U}}_{\mathrm{SS}_{\mathrm{s}}}, \quad r=1, \ldots, R
$$

with $\boldsymbol{\Gamma}^{(r)}=\boldsymbol{T} \boldsymbol{\Phi}^{(r)} \boldsymbol{T}^{-1}$. The $R$ unknown matrices $\boldsymbol{\Gamma}^{(r)} \in$ $\mathbb{C}^{d \times d}$ can be estimated, e.g., via least squares (LS), i.e.,

$$
\hat{\boldsymbol{\Gamma}}^{(r)}=\left(\tilde{\boldsymbol{J}}_{\mathrm{SS}_{1}}^{(r)} \hat{\boldsymbol{U}}_{\mathrm{SS}_{\mathrm{s}}}\right)^{+} \tilde{\boldsymbol{J}}_{\mathrm{SS}_{2}}^{(r)} \hat{\boldsymbol{U}}_{\mathrm{SS}_{\mathrm{s}}} \in \mathbb{C}^{d \times d} .
$$

Finally, after solving (19) for $\hat{\boldsymbol{\Gamma}}^{(r)}$ in each mode independently, the correctly paired spatial frequency estimates are given by $\hat{\mu}_{i}^{(r)}=\arg \left\{\hat{\lambda}_{i}^{(r)}\right\}, \quad i=1, \ldots, d$. The eigenvalues $\hat{\lambda}_{i}^{(r)}$ of $\hat{\boldsymbol{\Gamma}}^{(r)}$ are obtained by performing a joint eigendecomposition across all $R$ dimensions [39] or via the simultaneous Schur decomposition [7]. Alternatively, $R$-D Unitary ESPRIT [7] can be applied to estimate the $R \cdot d$ parameters, which is preferable due to its better performance at low SNRs and its real-valued implementation.

\section{B. R-D Spatial Smoothing for Strictly Non-Circular Sources}

If only NC sources are present, a modified spatial smoothing concept can be applied to the NC model in (10] [18], where we 
select $2 M_{\text {sub }}$ out of $2 M$ virtual sensors. Thus, the $L$ selection matrices in 13 are extended to

$$
\boldsymbol{J}_{\ell_{1}, \ldots, \ell_{R-1}, \ell_{R}}^{(\mathrm{nc})}=\boldsymbol{I}_{2} \otimes \boldsymbol{J}_{\ell_{1}, \ldots, \ell_{R-1}, \ell_{R}} \in \mathbb{R}^{2 M_{\mathrm{sub}} \times 2 M} .
$$

The resulting spatially smoothed data matrix $\boldsymbol{X}_{\mathrm{SS}}^{(\mathrm{nc})}$ of size $2 M_{\text {sub }} \times N L$ is then given by

$$
\begin{aligned}
\boldsymbol{X}_{\mathrm{SS}}^{(\mathrm{nc})} & =\left[\begin{array}{lll}
\boldsymbol{J}_{1, \cdots, 1,1}^{(\mathrm{nc})} \boldsymbol{X}^{(\mathrm{nc})} & \cdots & \boldsymbol{J}_{1, \cdots, 1, L_{r}}^{(\mathrm{nc})} \boldsymbol{X}^{(\mathrm{nc})} \\
\boldsymbol{J}_{1, \cdots, 2,1}^{(\mathrm{nc})} \boldsymbol{X}^{(\mathrm{nc})} & \cdots & \boldsymbol{J}_{L_{1}, \cdots, L_{R-1}, L_{R}}^{(\mathrm{nc})} \boldsymbol{X}^{(\mathrm{nc})}
\end{array}\right] .
\end{aligned}
$$

Following the lines of the previous subsection, we can compactly express 210 as

$$
\begin{aligned}
\boldsymbol{X}_{\mathrm{SS}}^{(\mathrm{nc})} & =\overline{\boldsymbol{A}}_{\mathrm{SS}}^{(\mathrm{nc})} \boldsymbol{\Phi}\left(\boldsymbol{I}_{L} \otimes \overline{\boldsymbol{S}}\right)+\boldsymbol{N}_{\mathrm{SS}}^{(\mathrm{nc})} \\
& =\boldsymbol{X}_{\mathrm{SS}}^{(\mathrm{nc})}+\boldsymbol{N}_{\mathrm{SS}}^{(\mathrm{nc})} \in \mathbb{C}^{2 M_{\mathrm{sub}} \times N L},
\end{aligned}
$$

where $\overline{\boldsymbol{A}}_{\mathrm{SS}}^{(\mathrm{nc})}=\boldsymbol{J}_{1, \cdots, 1,1}^{(\mathrm{nc})} \overline{\boldsymbol{A}}^{(\mathrm{nc})} \in \mathbb{C}^{2 M_{\mathrm{sub} \times d}}$ and $\boldsymbol{X}_{\mathrm{SS}_{0}}^{(\mathrm{nc})}$ is the unperturbed spatially smoothed $\mathrm{NC}$ data matrix. Note that spatial smoothing cannot be applied before $\boldsymbol{X}^{(\mathrm{nc})}$ is formed (10) as this would destroy the NC structure of the source signals.

As in the previous cases, $\boldsymbol{A}_{\mathrm{SS}}^{(\mathrm{nc})}$ is shift-invariant and satisfies

$$
\tilde{\boldsymbol{J}}_{\mathrm{SS}_{1}}^{(\mathrm{nc})(r)} \overline{\boldsymbol{A}}_{\mathrm{SS}}^{(\mathrm{nc})} \boldsymbol{\Phi}^{(r)}=\tilde{\boldsymbol{J}}_{\mathrm{SS}_{2}}^{(\mathrm{nc})(r)} \overline{\boldsymbol{A}}_{\mathrm{SS}}^{(\mathrm{nc})}, \quad r=1, \ldots, R,
$$

where $\tilde{\boldsymbol{J}}_{\mathrm{SS}_{k}}^{(\mathrm{nc})(r)} \in \mathbb{R}^{2 \frac{M_{\mathrm{sub}}}{M_{\mathrm{sub}}} M_{\mathrm{sub}_{r}}^{(\mathrm{sel})} \times 2 M_{\mathrm{sub}}}, k=1,2$ are the corresponding selection matrices that select $2 M_{\mathrm{sub}_{r}}^{(\mathrm{sel})}$ elements for the first and the second subarray in the $r$-th mode. They are defined as $\tilde{\boldsymbol{J}}_{\mathrm{SS}_{k}}^{(\mathrm{nc})(r)}=\boldsymbol{I}_{\prod_{l=1}^{r-1} M_{l}} \otimes \boldsymbol{J}_{\mathrm{SS}_{k}}^{(\mathrm{nc})(r)} \otimes \boldsymbol{I}_{\prod_{l=r+1}^{R} M_{l}}$, where $\boldsymbol{J}_{\mathrm{SS}_{k}}^{(\mathrm{nc})(r)}=\boldsymbol{I}_{2} \otimes \boldsymbol{J}_{\mathrm{SS}_{k}}^{(r)} \in \mathbb{R}^{2 M_{\mathrm{sub}_{r}}^{(\mathrm{sel})} \times 2 M_{\mathrm{sub}_{r}} \text { are the } r \text {-mode }}$ selection matrices for the first and second subarray. Again, $R$ D ESPRIT-type algorithms such as $R$-D NC Standard ESPRIT and $R$-D NC Unitary ESPRIT [19] can be used to estimate the $R \cdot d$ parameters.

\section{Performance of $R$-D ESPRIT-Type Algorithms WITH SPATIAL SMOOTHING}

In this section, we present first-order error expansions of $R$-D Standard ESPRIT and $R$-D Unitary ESPRIT both with spatial smoothing. The derived expressions rely on the data model (16) in Section III-A

\section{A. R-D Standard ESPRIT with Spatial Smoothing}

For the perturbation analysis of the estimation error, we adopt the analytical framework proposed in [9] along with its extension in [11]. The authors of [9] assume a small additive noise perturbation and derive an explicit first-order error expansion of the subspace estimation error in terms of the noise $\boldsymbol{N}$, which is followed by a corresponding expression for the parameter estimation error $\Delta \mu_{i}$. As a follow-up, analytical expressions for the MSE that only require a zero mean and finite SO moments of the noise have been derived in [11]. From (16), it is clear that these assumptions are not violated by spatial smoothing such that [9] and [11] are still applicable for the performance analysis.
To derive the signal subspace estimation error for (16), we express the SVD of the noise-free spatially smoothed observations $\boldsymbol{X}_{\mathrm{SS}_{0}}$ as

$$
\boldsymbol{X}_{\mathrm{SS}_{0}}=\left[\begin{array}{ll}
\boldsymbol{U}_{\mathrm{SS}_{\mathrm{s}}} & \boldsymbol{U}_{\mathrm{SS}_{\mathrm{n}}}
\end{array}\right]\left[\begin{array}{cc}
\boldsymbol{\Sigma}_{\mathrm{SS}_{\mathrm{s}}} & \mathbf{0} \\
\mathbf{0} & \mathbf{0}
\end{array}\right]\left[\begin{array}{ll}
\boldsymbol{V}_{\mathrm{SS}_{\mathrm{s}}} & \boldsymbol{V}_{\mathrm{SS}_{\mathrm{n}}}
\end{array}\right]^{\mathrm{H}},
$$

where $\boldsymbol{U}_{\mathrm{SS}_{\mathrm{s}}} \in \mathbb{C}^{M_{\mathrm{sub}} \times d}, \boldsymbol{U}_{\mathrm{SS}_{\mathrm{n}}} \in \mathbb{C}^{M_{\mathrm{sub}} \times(N L-d)}$, and $\boldsymbol{V}_{\mathrm{SS}_{\mathrm{s}}} \in$ $\mathbb{C}^{N L \times d}$ span the signal subspace, the noise subspace, and the row space, respectively, and $\boldsymbol{\Sigma}_{\mathrm{SS}_{\mathrm{s}}} \in \mathbb{R}^{d \times d}$ contains the nonzero singular values on its diagonal. Writing the perturbed signal subspace estimate $\hat{\boldsymbol{U}}_{\mathrm{SS}_{\mathrm{s}}}$ computed from the SVD of $\boldsymbol{X}_{\mathrm{SS}}$ as $\hat{\boldsymbol{U}}_{\mathrm{SS}_{\mathrm{s}}}=\boldsymbol{U}_{\mathrm{SS}_{\mathrm{s}}}+\Delta \boldsymbol{U}_{\mathrm{SS}_{\mathrm{s}}}$, where $\Delta \boldsymbol{U}_{\mathrm{SS}_{\mathrm{s}}}$ denotes the signal subspace error, the first-order approximation using [9] is given by

$$
\Delta \boldsymbol{U}_{\mathrm{SS}_{\mathrm{s}}}=\boldsymbol{U}_{\mathrm{SS}_{\mathrm{n}}} \boldsymbol{U}_{\mathrm{SS}_{\mathrm{n}}}^{\mathrm{H}} \boldsymbol{N}_{\mathrm{SS}} \boldsymbol{V}_{\mathrm{SS}_{\mathrm{s}}} \boldsymbol{\Sigma}_{\mathrm{SS}_{\mathrm{s}}}^{-1}+\mathcal{O}\left\{\nu^{2}\right\}
$$

where $\nu=\left\|N_{\mathrm{SS}}\right\|$, and $\|\cdot\|$ represents an arbitrary submultiplicative norm. For the estimation error $\Delta \mu_{i}^{(r)}$ of the $i$-th spatial frequency in the $r$-th mode obtained by the LS solution, we have [9]

$$
\begin{aligned}
\Delta \mu_{i}^{(r)}=\operatorname{Im}\left\{\boldsymbol{p}_{i}^{\mathrm{T}}\right. & \left(\tilde{\boldsymbol{J}}_{\mathrm{SS}_{1}}^{(r)} \boldsymbol{U}_{\mathrm{SS}_{\mathrm{s}}}\right)^{+}\left[\tilde{\boldsymbol{J}}_{\mathrm{SS}_{2}}^{(r)} / \lambda_{i}^{(r)}\right. \\
& \left.\left.-\tilde{\boldsymbol{J}}_{\mathrm{SS}_{1}}^{(r)}\right] \Delta \boldsymbol{U}_{\mathrm{SS}_{\mathrm{s}}} \boldsymbol{q}_{i}\right\}+\mathcal{O}\left\{\nu^{2}\right\},
\end{aligned}
$$

where $\lambda_{i}^{(r)}=\mathrm{e}^{\mathrm{j} \mu_{i}^{(r)}}$ is the $i$-th eigenvalue of $\boldsymbol{\Gamma}^{(r)}, \boldsymbol{q}_{i}$ represents the $i$-th eigenvector of $\boldsymbol{\Gamma}^{(r)}$ and the $i$-th column vector of the eigenvector matrix $\boldsymbol{Q}$, and $\boldsymbol{p}_{i}^{\mathrm{T}}$ is the $i$-th row vector of $\boldsymbol{P}=\boldsymbol{Q}^{-1}$. Hence, the eigendecomposition of $\boldsymbol{\Gamma}^{(r)}$ is given by $\boldsymbol{\Gamma}^{(r)}=\boldsymbol{Q} \boldsymbol{\Lambda}^{(r)} \boldsymbol{Q}^{-1}$, where $\boldsymbol{\Lambda}^{(r)}$ contains the eigenvalues $\lambda_{i}^{(r)}$ on its diagonal.

Finally, to compute the first-order MSE expression for $R$ D Standard ESPRIT with spatial smoothing, we extend the results in [11]. The MSE for the $i$-th spatial frequency in the $r$-th mode is given by

$$
\begin{aligned}
\mathbb{E}\left\{\left(\Delta \mu_{i}^{(r)}\right)^{2}\right\} \approx \frac{1}{2} & \left(\boldsymbol{r}_{\mathrm{SS}_{i}}^{(r)^{\mathrm{H}}} \boldsymbol{W}_{\mathrm{SS}}^{*} \boldsymbol{R}_{\mathrm{SS}}^{\mathrm{T}} \boldsymbol{W}_{\mathrm{SS}}^{\mathrm{T}} \boldsymbol{r}_{\mathrm{SS}_{i}}^{(r)}\right. \\
& \left.-\operatorname{Re}\left\{\boldsymbol{r}_{\mathrm{SS}_{i}}^{(r)^{\mathrm{T}}} \boldsymbol{W}_{\mathrm{SS}} \boldsymbol{C}_{\mathrm{SS}}^{\mathrm{T}} \boldsymbol{W}_{\mathrm{SS}^{\mathrm{T}}}^{\mathrm{T}} \boldsymbol{r}_{\mathrm{SS}_{i}}^{(r)}\right\}\right)
\end{aligned}
$$

where

$$
\begin{gathered}
\boldsymbol{r}_{\mathrm{SS}_{i}}^{(r)}=\boldsymbol{q}_{i} \otimes\left(\left[\left(\tilde{\boldsymbol{J}}_{\mathrm{SS}_{1}}^{(r)} \boldsymbol{U}_{\mathrm{SS}_{\mathrm{s}}}\right)^{+}\left(\tilde{\boldsymbol{J}}_{\mathrm{SS}_{2}}^{(r)} / \lambda_{i}^{(r)}-\tilde{\boldsymbol{J}}_{\mathrm{SS}_{1}}^{(r)}\right)\right]^{\mathrm{T}} \boldsymbol{p}_{i}\right), \\
\boldsymbol{W}_{\mathrm{SS}}=\left(\boldsymbol{\Sigma}_{\mathrm{SS}_{\mathrm{s}}}^{-1} \boldsymbol{V}_{\mathrm{SS}_{\mathrm{s}}}^{\mathrm{T}}\right) \otimes\left(\boldsymbol{U}_{\mathrm{SS}_{\mathrm{n}}} \boldsymbol{U}_{\mathrm{SS}_{\mathrm{n}}}^{\mathrm{H}}\right) \in \mathbb{C}^{M_{\mathrm{sub}} d \times M_{\mathrm{sub}} N L}
\end{gathered}
$$

In order to apply (27), we require the covariance matrix $\boldsymbol{R}_{\mathrm{SS}}=$ $\mathbb{E}\left\{\boldsymbol{n}_{\mathrm{SS}} \boldsymbol{n}_{\mathrm{SS}}^{\mathrm{H}}\right\} \in \mathbb{C}^{M_{\mathrm{sub}} N L \times M_{\mathrm{sub}} N L}$ and the pseudo-covariance matrix $\stackrel{C}{\mathrm{SS}}=\mathbb{E}\left\{\boldsymbol{n}_{\mathrm{SS}} \boldsymbol{n}_{\mathrm{SS}}^{\mathrm{T}}\right\} \in \mathbb{C}^{M_{\mathrm{sub}} N L \times M_{\mathrm{sub}} N L}$ of the spatially smoothed noise $\boldsymbol{n}_{\mathrm{SS}}=\operatorname{vec}\left\{\boldsymbol{N}_{\mathrm{SS}}\right\} \in \mathbb{C}^{M_{\text {sub }} N L \times 1}$. It is clear that the preprocessing via spatial smoothing modifies the prior noise statistics, resulting in colored noise. However,

\footnotetext{
${ }^{2}$ A matrix norm is called sub-multiplicative if $\|\boldsymbol{A} \cdot \boldsymbol{B}\| \leq\|\boldsymbol{A}\| \cdot\|\boldsymbol{B}\|$ for arbitrary matrices $\boldsymbol{A}$ and $\boldsymbol{B}$.
} 
in what follows, we analytically derive the SO noise statistics of the spatially smoothed noise. We first expand $\boldsymbol{n}_{\mathrm{SS}}$ as

$$
\begin{aligned}
\boldsymbol{n}_{\mathrm{SS}} & =\operatorname{vec}\left\{\left[\begin{array}{cc}
{\left[\boldsymbol{J}_{1, \cdots, 1,1} \boldsymbol{N}\right.} & \cdots
\end{array} \boldsymbol{J}_{L_{1}, \cdots, L_{R-1}, L_{R}} \boldsymbol{N}\right]\right\} \\
& =\left[\begin{array}{c}
\left(\boldsymbol{I}_{N} \otimes \boldsymbol{J}_{1, \cdots, 1,1}\right) \\
\vdots \\
\left(\boldsymbol{I}_{N} \otimes \boldsymbol{J}_{L_{1}, \cdots, L_{R-1}, L_{R}}\right)
\end{array}\right] \cdot \boldsymbol{n}=\boldsymbol{M} \cdot \boldsymbol{n},
\end{aligned}
$$

where $\boldsymbol{M} \in \mathbb{R}^{M_{\text {sub }} N L \times M N}, \boldsymbol{n}=\operatorname{vec}\{\boldsymbol{N}\} \in \mathbb{C}^{M N \times 1}$ is the unsmoothed noise component, and we have used the property vec $\{\boldsymbol{A} \boldsymbol{X} \boldsymbol{B}\}=\left(\boldsymbol{B}^{\mathrm{T}} \otimes \boldsymbol{A}\right) \operatorname{vec}\{\boldsymbol{X}\}$ for arbitrary matrices $\boldsymbol{A}, \boldsymbol{B}$, and $\boldsymbol{X}$ of appropriate sizes. Thus, the SO statistics of $\boldsymbol{n}_{\mathrm{SS}}$ can be expressed in terms of the covariance matrix $\boldsymbol{R}_{\mathrm{nn}}=\mathbb{E}\left\{\boldsymbol{n} \boldsymbol{n}^{\mathrm{H}}\right\} \in \mathbb{C}^{M N \times M N}$ and the pseudo-covariance matrix $\boldsymbol{C}_{\mathrm{nn}}=\mathbb{E}\left\{\boldsymbol{n} \boldsymbol{n}^{\mathrm{T}}\right\} \in \mathbb{C}^{M N \times M N}$ of $\boldsymbol{n}$. We obtain

$$
\boldsymbol{R}_{\mathrm{SS}}=\boldsymbol{M} \boldsymbol{R}_{\mathrm{nn}} \boldsymbol{M}^{\mathrm{T}}, \quad \boldsymbol{C}_{\mathrm{SS}}=\boldsymbol{M} \boldsymbol{C}_{\mathrm{nn}} \boldsymbol{M}^{\mathrm{T}} .
$$

\section{B. R-D Unitary ESPRIT with Spatial Smoothing}

It was shown in [11] that the asymptotic performance of $R$ D Unitary-ESPRIT is found once forward-backward-averaging (FBA) is taken into account. FBA is performed by replacing the spatially smoothed data matrix $\boldsymbol{X}_{\mathrm{SS}} \in \mathbb{C}^{M_{\mathrm{sub}} \times N L}$ by the column-augmented data matrix $\tilde{\boldsymbol{X}}_{\mathrm{SS}} \in \mathbb{C}^{M_{\text {sub }} \times 2 N L}$ defined by

$$
\tilde{\boldsymbol{X}}_{\mathrm{SS}}=\left[\begin{array}{ll}
\boldsymbol{X}_{\mathrm{SS}} & \boldsymbol{\Pi}_{M_{\mathrm{sub}}} \boldsymbol{X}_{\mathrm{SS}}^{*} \boldsymbol{\Pi}_{N L}
\end{array}\right]=\tilde{\boldsymbol{X}}_{\mathrm{SS}_{0}}+\tilde{\boldsymbol{N}}_{\mathrm{SS}},
$$

where $\tilde{\boldsymbol{X}}_{\mathrm{SS}_{0}}$ is the noiseless FBA-processed spatially smoothed data matrix. Following the steps of the previous subsection, the first-order MSE expression for $R$-D Unitary ESPRIT with spatial smoothing for the $i$-th spatial frequency in the $r$-th mode is given by

$$
\begin{aligned}
\mathbb{E}\left\{\left(\Delta \mu_{i}^{(r)}\right)^{2}\right\} \approx \frac{1}{2} & \left(\tilde{\boldsymbol{r}}_{\mathrm{SS}_{i}}^{(r)} \tilde{\boldsymbol{W}}_{\mathrm{SS}}^{*} \tilde{\boldsymbol{R}}_{\mathrm{SS}}^{\mathrm{T}} \tilde{\boldsymbol{W}}_{\mathrm{SS}}^{\mathrm{T}} \tilde{\boldsymbol{r}}_{\mathrm{SS}_{i}}^{(r)}\right. \\
& \left.-\operatorname{Re}\left\{\tilde{\boldsymbol{r}}_{\mathrm{SS}_{i}}^{(r)^{\mathrm{T}}} \tilde{\boldsymbol{W}}_{\mathrm{SS}} \tilde{\boldsymbol{C}}_{\mathrm{SS}}^{\mathrm{T}} \tilde{\boldsymbol{W}}_{\mathrm{SS}}^{\mathrm{T}} \tilde{\boldsymbol{r}}_{\mathrm{SS}_{i}}^{(r)}\right\}\right)
\end{aligned}
$$

with

$$
\begin{aligned}
& \tilde{\boldsymbol{r}}_{\mathrm{SS}_{i}}^{(r)}=\tilde{\boldsymbol{q}}_{i} \otimes\left(\left[\left(\tilde{\boldsymbol{J}}_{\mathrm{SS}_{1}}^{(r)} \tilde{\boldsymbol{U}}_{\mathrm{SS}_{\mathrm{s}}}\right)^{+}\left(\tilde{\boldsymbol{J}}_{\mathrm{SS}_{2}}^{(r)} / \lambda_{i}^{(r)}-\tilde{\boldsymbol{J}}_{\mathrm{SS}_{1}}^{(r)}\right)\right]^{\mathrm{T}} \tilde{\boldsymbol{p}}_{i}\right), \\
& \tilde{\boldsymbol{W}}_{\mathrm{SS}}=\left(\tilde{\boldsymbol{\Sigma}}_{\mathrm{SS}_{\mathrm{s}}}^{-1} \tilde{\boldsymbol{V}}_{\mathrm{SS}_{\mathrm{s}}}^{\mathrm{T}}\right) \otimes\left(\tilde{\boldsymbol{U}}_{\mathrm{SS}_{\mathrm{n}}} \tilde{\boldsymbol{U}}_{\mathrm{SS}_{\mathrm{n}}}^{\mathrm{H}}\right) \in \mathbb{C}^{M_{\mathrm{sub}} d \times 2 M_{\mathrm{sub}} N L},
\end{aligned}
$$

where we have replaced the noise-free subspaces of $\boldsymbol{X}_{\mathrm{SS}_{0}}$ in (27) by the corresponding subspaces of $\tilde{\boldsymbol{X}}_{\mathrm{SS}_{0}}$, and $\boldsymbol{p}_{i}$ and $\boldsymbol{q}_{i}$ by $\tilde{\boldsymbol{p}}_{i}$ and $\tilde{\boldsymbol{q}}_{i}$, respectively. It can be shown that $\tilde{\boldsymbol{n}}_{\mathrm{SS}}=$ $\operatorname{vec}\left\{\tilde{\boldsymbol{N}}_{\mathrm{SS}}\right\} \in \mathbb{C}^{2 M_{\mathrm{sub}} N L \times 1}$ is given by

$$
\begin{aligned}
\tilde{\boldsymbol{n}}_{\mathrm{SS}} & =\operatorname{vec}\left\{\left[\begin{array}{ll}
\boldsymbol{N}_{\mathrm{SS}} & \boldsymbol{\Pi}_{M_{\mathrm{sub}}} \boldsymbol{N}_{\mathrm{SS}}^{*} \boldsymbol{\Pi}_{N L}
\end{array}\right]\right\} \\
& =\left[\begin{array}{c}
\operatorname{vec}\left\{\boldsymbol{N}_{\mathrm{SS}}\right\} \\
\operatorname{vec}\left\{\boldsymbol{\Pi}_{M_{\mathrm{sub}}} \boldsymbol{N}_{\mathrm{SS}}^{*} \boldsymbol{\Pi}_{N L}\right\}
\end{array}\right]=\left[\begin{array}{c}
\boldsymbol{n}_{\mathrm{SS}} \\
\boldsymbol{\Pi}_{M_{\mathrm{sub}} N L} \boldsymbol{n}_{\mathrm{SS}}^{*}
\end{array}\right] .
\end{aligned}
$$

Therefore, the expressions for $\tilde{\boldsymbol{R}}_{\mathrm{SS}}=\mathbb{E}\left\{\tilde{\boldsymbol{n}}_{\mathrm{SS}} \tilde{\boldsymbol{n}}_{\mathrm{SS}}^{\mathrm{H}}\right\} \in$ $\mathbb{C}^{2 M_{\mathrm{sub}} N L \times 2 M_{\mathrm{sub}} N L}$ and $\tilde{\boldsymbol{C}}_{\mathrm{SS}}=\mathbb{E}\left\{\tilde{\boldsymbol{n}}_{\mathrm{SS}} \tilde{\boldsymbol{n}}_{\mathrm{SS}}^{\mathrm{T}}\right\} \in$ $\mathbb{C}^{2 M_{\text {sub }} N L \times 2 M_{\text {sub }} N L}$ can be derived in terms of 29

as

$$
\tilde{\boldsymbol{R}}_{\mathrm{SS}}=\boldsymbol{P}\left[\begin{array}{ll}
\boldsymbol{R}_{\mathrm{SS}} & \boldsymbol{C}_{\mathrm{SS}} \\
\boldsymbol{C}_{\mathrm{SS}}^{*} & \boldsymbol{R}_{\mathrm{SS}}^{*}
\end{array}\right] \boldsymbol{P}^{\mathrm{T}}, \quad \tilde{\boldsymbol{C}}_{\mathrm{SS}}=\boldsymbol{P}\left[\begin{array}{ll}
\boldsymbol{C}_{\mathrm{SS}} & \boldsymbol{R}_{\mathrm{SS}} \\
\boldsymbol{R}_{\mathrm{SS}}^{*} & \boldsymbol{C}_{\mathrm{SS}}^{*}
\end{array}\right] \boldsymbol{P}^{\mathrm{T}},
$$

where $\boldsymbol{P}=\operatorname{blkdiag}\left\{\boldsymbol{I}_{M_{\mathrm{sub}} N L}, \boldsymbol{\Pi}_{M_{\mathrm{sub}} N L}\right\}$.

\section{PERformance of $R$-D NC ESPRIT-Type AlgorithmS WITH SPATIAL SMOOTHING}

In this section, we derive first-order analytical error expressions of $R$-D NC Standard ESPRIT and $R$-D NC Unitary ESPRIT for strictly non-circular sources both with spatial smoothing. As will be shown in Subsection $\mathrm{V}$-B the performance of both algorithms is asymptotically identical in the high effective SNR. Therefore, we first resort to the simpler derivation for the spatially smoothed $R$-D NC Standard ESPRIT algorithm and then show its equivalence to the spatially smoothed $R$-D NC Unitary ESPRIT algorithm. Our results are based on the data model 22 in Section III-B

\section{A. R-D NC Standard ESPRIT with Spatial Smoothing}

In [19], we have shown that the framework of [9] is still applicable to the augmented measurement matrix $\boldsymbol{X}^{(\mathrm{nc})}$ obtained by the preprocessing scheme for non-circular sources. From (22), it is apparent that adding spatial smoothing as a second preprocessing step does not violate the assumptions, such that the steps from Section IV-A can be applied to the spatially smoothed augmented data matrix $\boldsymbol{X}_{\mathrm{SS}}^{(\mathrm{nc})}$.

As a result, equivalently to 27, the first-order MSE expression for $R$-D NC Standard ESPRIT with spatial smoothing for the $i$-th spatial frequency in the $r$-th mode is given by

$$
\begin{array}{r}
\mathbb{E}\left\{\left(\Delta \mu_{i}^{(r)}\right)^{2}\right\} \approx \frac{1}{2}\left(\boldsymbol{r}_{\mathrm{SS}_{i}}^{(\mathrm{nc})(r)^{\mathrm{H}}} \boldsymbol{W}_{\mathrm{SS}}^{(\mathrm{nc})^{*}} \boldsymbol{R}_{\mathrm{SS}}^{(\mathrm{nc})^{\mathrm{T}}} \boldsymbol{W}_{\mathrm{SS}}^{(\mathrm{nc})^{\mathrm{T}}} \boldsymbol{r}_{\mathrm{SS}_{i}}^{(\mathrm{nc})(r)}\right. \\
-\operatorname{Re}\left\{\boldsymbol{r}_{\mathrm{SS}_{i}}^{(\mathrm{nc})(r)^{\mathrm{T}}} \boldsymbol{W}_{\mathrm{SS}}^{(\mathrm{nc})} \boldsymbol{C}_{\mathrm{SS}}^{(\mathrm{nc})^{\mathrm{T}}} \boldsymbol{W}_{\left.\left.\mathrm{SS}^{(\mathrm{nc})^{\mathrm{T}}} \boldsymbol{r}_{\mathrm{SS}_{i}}^{(\mathrm{nc})(r)}\right\}\right),}^{(33)}\right.
\end{array}
$$

where

$$
\begin{aligned}
& \tilde{\boldsymbol{r}}_{\mathrm{SS}_{i}}^{(\mathrm{nc})(r)}=\boldsymbol{q}_{i}^{(\mathrm{nc})} \otimes\left(\left[\left(\tilde{\boldsymbol{J}}_{\mathrm{SS}_{1}(\mathrm{nc})(r)} \boldsymbol{U}_{\mathrm{SS}_{\mathrm{s}}}^{(\mathrm{nc})}\right)^{+}\right.\right. \\
& \left.\left.\cdot\left(\tilde{\boldsymbol{J}}_{\mathrm{SS}_{2}}^{(\mathrm{nc})(r)} / \lambda_{i}^{(r)}-\tilde{\boldsymbol{J}}_{\mathrm{SS}_{1}}^{(\mathrm{nc})(r)}\right)\right]^{\mathrm{T}} \boldsymbol{p}_{i}^{(\mathrm{nc})}\right) \in \mathbb{C}^{2 M_{\mathrm{sub}} d \times 1}, \\
& \boldsymbol{W}_{\mathrm{SS}}^{(\mathrm{nc})}=\left(\boldsymbol{\Sigma}_{\mathrm{SS}_{\mathrm{s}}}^{(\mathrm{nc})^{-1}} \boldsymbol{V}_{\mathrm{SS}_{\mathrm{s}}}^{(\mathrm{nc})^{\mathrm{T}}}\right) \otimes\left(\boldsymbol{U}_{\mathrm{SS}_{\mathrm{n}}}^{(\mathrm{nc})} \boldsymbol{U}_{\mathrm{SS}_{\mathrm{n}}}^{\left(\mathrm{nc}_{\mathrm{n}}^{\mathrm{H}}\right.}\right) \in \mathbb{C}^{2 M_{\mathrm{sub}} d \times 2 M_{\mathrm{sub}} N L},
\end{aligned}
$$

where $\boldsymbol{p}_{i}^{(\mathrm{nc})}$ and $\boldsymbol{q}_{i}^{(\mathrm{nc})}$ replace $\boldsymbol{p}_{i}$ and $\boldsymbol{q}_{i}$, respectively, we have used the corresponding subspaces of $\boldsymbol{X}_{\mathrm{SS}_{0}}^{(\mathrm{nc})}$ defined in (22), and the selection matrices $\tilde{\boldsymbol{J}}_{\mathrm{SS}_{k}}^{(\mathrm{nc})(r)}, k=1,2$, are given in (23).

The spatially smoothed augmented noise contribution $\boldsymbol{n}_{\mathrm{SS}}^{(\mathrm{nc})}=\operatorname{vec}\left\{\boldsymbol{N}_{\mathrm{SS}}^{(\mathrm{nc})}\right\} \in \mathbb{C}^{2 M_{\mathrm{sub}} N L \times 1}$ can be expressed similarly to 28 as

$$
\begin{aligned}
& \boldsymbol{n}_{\mathrm{SS}}^{(\mathrm{nc})}=\operatorname{vec}\left\{\left[\begin{array}{lll}
\boldsymbol{J}_{1, \cdots, 1,1}^{(\mathrm{nc})} \boldsymbol{N}^{(\mathrm{nc})} & \ldots & \boldsymbol{J}_{L_{1}, \cdots, L_{R-1}, L_{R}}^{(\mathrm{nc})} \boldsymbol{N}^{(\mathrm{nc})}
\end{array}\right]\right\} \\
& =\left[\begin{array}{c}
\left(\boldsymbol{I}_{N} \otimes \boldsymbol{J}_{1, \cdots, 1,1}^{(\mathrm{nc})}\right) \\
\vdots \\
\left(\boldsymbol{I}_{N} \otimes \boldsymbol{J}_{L_{1}, \cdots, L_{R-1}, L_{R}}^{(\mathrm{nc})}\right.
\end{array}\right] \cdot \boldsymbol{n}^{(\mathrm{nc})}=\boldsymbol{M}^{(\mathrm{nc})} \cdot \boldsymbol{n}^{(\mathrm{nc})},
\end{aligned}
$$

where $\boldsymbol{M}^{(\mathrm{nc})} \in \mathbb{R}^{2 M_{\mathrm{sub}} N L \times 2 M N}$ and $\boldsymbol{n}^{(\mathrm{nc})}=\operatorname{vec}\left\{\boldsymbol{N}^{(\mathrm{nc})}\right\} \in$ $\mathbb{C}^{2 M N \times 1}$. Note that we have shown in [19] that $\boldsymbol{n}^{(\mathrm{nc})}$ can be represented as

$$
\boldsymbol{n}^{(\mathrm{nc})}=\tilde{\boldsymbol{K}} \cdot\left[\begin{array}{c}
\boldsymbol{n} \\
\boldsymbol{n}^{*}
\end{array}\right]
$$


where $\tilde{\boldsymbol{K}}=\boldsymbol{K}_{2 M, N}^{\mathrm{T}} \cdot \operatorname{blkdiag}\left\{\boldsymbol{K}_{M, N}, \boldsymbol{K}_{M, N} \cdot\left(\boldsymbol{I}_{N} \otimes \boldsymbol{\Pi}_{M}\right)\right\}$ and $\boldsymbol{K}_{M, N} \in \mathbb{R}^{M N \times M N}$ is the commutation matrix that satisfies $\boldsymbol{K}_{M, N} \cdot \operatorname{vec}\{\boldsymbol{A}\}=\operatorname{vec}\left\{\boldsymbol{A}^{\mathrm{T}}\right\}$ for arbitrary matrices $\boldsymbol{A} \in \mathbb{C}^{M \times N}$ [40]. Then, $\boldsymbol{R}_{\mathrm{SS}}^{(\mathrm{nc})}=\mathbb{E}\left\{\boldsymbol{n}_{\mathrm{SS}}^{(\mathrm{nc})} \boldsymbol{n}_{\mathrm{SS}}^{(\mathrm{nc})^{\mathrm{H}}}\right\} \in$ $\mathbb{C}^{2 M_{\mathrm{sub}} N L \times 2 M_{\mathrm{sub}} N L}$ and $\boldsymbol{C}_{\mathrm{SS}}^{(\mathrm{nc})}=\mathbb{E}\left\{\boldsymbol{n}_{\mathrm{SS}}^{(\mathrm{nc})} \boldsymbol{n}_{\mathrm{SS}}^{(\mathrm{nc})^{\mathrm{T}}}\right\} \in$ $\mathbb{C}^{2 M_{\text {sub }} N L \times 2 M_{\text {sub }} N L}$ can be computed as

$\boldsymbol{R}_{\mathrm{SS}}^{(\mathrm{nc})}=\boldsymbol{M}^{(\mathrm{nc})} \boldsymbol{R}_{\mathrm{nn}}^{(\mathrm{nc})} \boldsymbol{M}^{(\mathrm{nc})^{\mathrm{T}}}, \quad \boldsymbol{C}_{\mathrm{SS}}^{(\mathrm{nc})}=\boldsymbol{M}^{(\mathrm{nc})} \boldsymbol{C}_{\mathrm{nn}}^{(\mathrm{nc})} \boldsymbol{M}^{(\mathrm{nc})^{\mathrm{T}}}$

where $\boldsymbol{R}_{\mathrm{nn}}^{(\mathrm{nc})} \in \mathbb{C}^{2 M N \times 2 M N}$ and $\boldsymbol{C}_{\mathrm{nn}}^{(\mathrm{nc})} \in \mathbb{C}^{2 M N \times 2 M N}$ are given by [19]

$$
\begin{aligned}
& \boldsymbol{R}_{\mathrm{nn}}^{(\mathrm{nc})}=\mathbb{E}\left\{\boldsymbol{n}^{(\mathrm{nc})} \boldsymbol{n}^{\left.(\mathrm{nc})^{\mathrm{H}}\right\}}=\tilde{\boldsymbol{K}}\left[\begin{array}{ll}
\boldsymbol{R}_{\mathrm{nn}} & \boldsymbol{C}_{\mathrm{nn}} \\
\boldsymbol{C}_{\mathrm{nn}}^{*} & \boldsymbol{R}_{\mathrm{nn}}^{*}
\end{array}\right] \tilde{\boldsymbol{K}}^{\mathrm{T}},\right. \\
& \boldsymbol{C}_{\mathrm{nn}}^{(\mathrm{nc})}=\mathbb{E}\left\{\boldsymbol{n}^{(\mathrm{nc})} \boldsymbol{n}^{\left.(\mathrm{nc})^{\mathrm{T}}\right\}}=\tilde{\boldsymbol{K}}\left[\begin{array}{ll}
\boldsymbol{C}_{\mathrm{nn}} & \boldsymbol{R}_{\mathrm{nn}} \\
\boldsymbol{R}_{\mathrm{nn}}^{*} & \boldsymbol{C}_{\mathrm{nn}}^{*}
\end{array}\right] \tilde{\boldsymbol{K}}^{\mathrm{T}} .\right.
\end{aligned}
$$

\section{B. R-D NC Unitary ESPRIT with Spatial Smoothing}

We have shown in [19] that $R$-D NC Standard ESPRIT and $R$-D NC Unitary ESPRIT both have the same asymptotic performance in the high effective SNR regime. It was established that applying FBA to the augmented matrix $\boldsymbol{X}^{(\mathrm{nc})}$ does not improve the signal subspace estimate and that the real-valued transformation has no effect on the asymptotic performance in the high effective SNR. In this subsection, we prove that these properties still hold when spatial smoothing is applied to both algorithms. To this end, we first investigate the effect of FBA and state the following theorem:

Theorem 1. Applying FBA to $\boldsymbol{X}_{\mathrm{SS}}^{(\mathrm{nc})}$ does not improve the signal subspace estimate.

Proof: The proof is given in Appendix A.

Next, we analyze the real-valued transformation as the second preprocessing step of $R$-D NC Unitary ESPRIT with spatial smoothing and formulate the theorem:

Theorem 2. The spatially smoothed R-D NC Unitary ESPRIT algorithm and the spatially smoothed R-D NC Standard ESPRIT algorithm with FBA preprocessing perform asymptotically identical in the high effective SNR.

Proof: The proof of this theorem follows the same steps as the one for the case without spatial smoothing considered in [19]. This is due to the fact that spatial smoothing modifies the NC signal subspace of $R$-D NC Standard ESPRIT and $R$-D NC Unitary ESPRIT in the same way.

As a result of Theorem 1 and Theorem 2 , we can conclude that the asymptotic performance of $R$-D NC Standard ESPRIT and $R$-D NC Unitary ESPRIT both with spatial smoothing is identical in the high effective SNR.

\section{Single Source CASE}

The derived analytical MSE expressions for the $R$-D ESPRIT-type methods with spatial smoothing are deterministic and formulated in terms of the subspaces of the noisefree observations. In [10] and [19], we have considered the special case of a single source for $R$-D ESPRIT-type methods without spatial smoothing to gain explicit insights into how the MSE expressions depend on the physical parameters, e.g., the number of sensors $M$, the sample size $N$, and the SNR. The knowledge of how the MSE expressions depend on these parameters can be of practical significance. For instance, this enables an objective comparison of different estimators or facilitates array design decisions on the value of $M$ required to achieve a target MSE for a specific SNR. Note that establishing general MSE expressions for an arbitrary number of sources is challenging given the complex dependence of the subspaces on the physical parameters. For the single source case, it was proven in [10] and [19] that neither FBA nor NC preprocessing can improve the MSE. However, in this section, we show that a significant gain can be achieved for the MSE of $R$ D ESPRIT-type methods for a single source when spatial smoothing is applied. Assuming an $R$-D uniform sampling grid, i.e., a ULA in each mode, and circularly symmetric white noise, we simplify the derived MSE expressions in 27), 31, and 33 for this special case. The result depends on the number of subarrays $L_{r}$ in the $r$-th mode as a design parameter, which we analytically compute in the $R$-D case by minimizing the MSE. It should be emphasized that these results for the special case $R=1$ are in line with those derived in [32]-[34] for harmonic retrieval. Here, the $R$-D extension is provided. Based on our $R$-D results, we explicitly compute the asymptotic spatial smoothing gain for arbitrary $R$ and the asymptotic efficiency for $R=1$ in closed-form.

\section{A. R-D ESPRIT-type Algorithms with Spatial Smoothing}

The final result for the simplified MSE expressions is summarized in the following theorem:

Theorem 3. For the case of an $M$-element $R$-D uniform sampling grid with an $M_{r}$-element ULA in the $r$-th mode, a single source $(d=1)$, and circularly symmetric white noise, the MSE in the $r$-th mode of R-D Standard ESPRIT and R-D Unitary ESPRIT with spatial smoothing as well as the MSE in the $r$-th mode of $R-D N C$ Standard ESPRIT and $R-D N C$ Unitary ESPRIT with spatial smoothing for a single source are given by $\mathrm{MSE}_{\mathrm{SS}}^{(r)}=\mathbb{E}\left\{\left(\Delta \mu^{(r)}\right)^{2}\right\}$, yielding

$$
\operatorname{MSE}_{\mathrm{SS}}^{(r)} \approx \begin{cases}\frac{1}{\hat{\rho}} \cdot \frac{1}{\left(M_{r}-L_{r}\right)^{2} L_{r}} \cdot \prod_{\substack{p=1 \\ p=r}}^{R} \frac{c_{p}}{M_{\mathrm{sub}_{p}}^{2} L_{p}^{2}} & \text { if } L_{r} \leq \frac{M_{r}}{2} \\ \frac{1}{\hat{\rho}} \cdot \frac{1}{\left(M_{r}-L_{r}\right) L_{r}^{2}} \cdot \prod_{\substack{p=1 \\ p \neq p}}^{R} \frac{c_{p}}{M_{\mathrm{sub}_{p}}^{2} L_{p}^{2}} & \text { if } L_{r}>\frac{M_{r}}{2},\end{cases}
$$

where $c_{p}$ is given in (40) and $\hat{\rho}$ represents the effective SNR $\hat{\rho}=N \hat{P}_{\mathrm{s}} / \sigma_{\mathrm{n}}^{2}$ with $\hat{P}_{\mathrm{s}}$ being the empirical source power given by $\hat{P}_{\mathrm{s}}=\|s\|_{2}^{2} / N$ and $s \in \mathbb{C}^{N \times 1}$.

Proof: See Appendix B.

Note that (39) as a function of $L_{r}$ is symmetric with respect to $L_{r}=M_{r} / 2$. In the special case of $R=1$, where $M_{r}=M$ and $L_{r}=L$, the MSE in 39] simplifies to

$$
\operatorname{MSE}_{\mathrm{SS}} \approx \begin{cases}\frac{1}{\hat{\rho}} \cdot \frac{1}{(M-L)^{2} L} & \text { if } L \leq \frac{M}{2} \\ \frac{1}{\hat{\rho}} \cdot \frac{1}{(M-L) L^{2}} & \text { if } L>\frac{M}{2} .\end{cases}
$$

Interestingly, we arrive at the same result for the MSE of all the considered spatially smoothed $R$-D ESPRIT-type algorithms for a single source, i.e., no additional gain from FBA or NC preprocessing can be achieved. 


\section{B. Optimal Number of Subarrays for Spatial Smoothing}

In the MSE expression in 39, the number of subarrays $L_{r}$ in each mode is a design parameter that can be optimized. Therefore, minimizing the MSE expression (39) with respect to $L_{r}$, yield 3

$$
L_{r}^{\mathrm{opt}}= \begin{cases}\frac{1}{3} \cdot M_{r} & \text { if } L_{r} \leq \frac{M_{r}}{2} \\ \frac{2}{3} \cdot M_{r} & \text { if } L_{r}>\frac{M_{r}}{2}\end{cases}
$$

where it is assumed that $M_{r}$ is a multiple of 3. A short proof is provided in Appendix C If $M_{r}$ is not a multiple of 3, we round to the nearest integer. Then, $L_{r}^{\mathrm{opt}}$ for the case $L_{r} \leq \frac{M_{r}}{2}$, for instance, is given by

$$
L_{r}^{\mathrm{opt}}= \begin{cases}\frac{1}{3} \cdot\left(M_{r}-1\right) & \text { if } M_{r} \bmod 3=1 \\ \frac{1}{3} \cdot\left(M_{r}+1\right) & \text { if } M_{r} \bmod 3=2\end{cases}
$$

It is worth highlighting that $L_{r}^{\mathrm{opt}}$ is independent of $L_{p}$ and $M_{p}$ for $p \neq r$, which is due to the separability of the array. Inserting $L_{r}^{\mathrm{opt}}$ from (42) and (43) into expression (39), we obtain $\mathrm{MSE}_{\mathrm{SS}_{\mathrm{opt}}}^{(r)}=\operatorname{MSE}_{\mathrm{SS}}^{(r)}\left(L_{r}^{\mathrm{opt}}\right)$ as

$$
\mathrm{MSE}_{\mathrm{SS}}^{(r)} \approx \begin{cases}\frac{1}{\hat{\rho}} \cdot \frac{27}{4} \cdot \frac{a}{M_{r}^{3}} & \text { if } M_{r} \bmod 3=0 \\ \frac{1}{\hat{\rho}} \cdot \frac{27}{4} \cdot \frac{a}{\left(M_{r}+\frac{1}{2}\right)^{2}\left(M_{r}-1\right)} & \text { if } M_{r} \bmod 3=1 \\ \frac{1}{\hat{\rho}} \cdot \frac{27}{4} \cdot \frac{a}{\left(M_{r}-\frac{1}{2}\right)^{2}\left(M_{r}+1\right)} & \text { if } M_{r} \bmod 3=2,\end{cases}
$$

where $a=\prod_{\substack{p=1 \\ p \neq r}}^{R} \frac{c_{p}}{M_{\mathrm{sub}_{p}}^{2} L_{p}^{2}}$. It is clear that the MSE for a fixed $\hat{\rho}$ is lowest when $M_{r}$ is a multiple of 3 . Again, for $R=1$, these results are in line with those derived in [32]-[34] for harmonic retrieval.

\section{Asymptotic Spatial Smoothing Gain}

Based on the result for $L_{r}^{\mathrm{opt}}$, the maximum asymptotic gain obtained from spatial smoothing can be explicitly quantified. To this end, we contrast $\operatorname{MSE}_{\mathrm{SS}}^{(r)}\left(L_{r}^{\mathrm{opt}}\right)$ from above with the result $\mathrm{MSE}^{(r)}=\frac{1}{\hat{\rho}} \cdot \frac{M_{r}}{M\left(M_{r}-1\right)^{2}}$ from [10] and [19] without spatial smoothing. The maximum asymptotic spatial smoothing gain in the $r$-th mode defined as $\eta_{\mathrm{SS}}^{(r)}\left(L_{r}^{\mathrm{opt}}\right)=$ $\mathrm{MSE}^{(r)} / \mathrm{MSE}_{\mathrm{SS}}^{(r)}\left(L_{r}^{\mathrm{opt}}\right)$ can be computed as

$$
\eta_{\mathrm{SS}}^{(r)}\left(L_{r}^{\mathrm{opt}}\right) \approx \begin{cases}\frac{4}{27} \cdot \frac{M_{r}^{4}}{\left(M_{r}-1\right)^{2}} \cdot \frac{1}{M a} & \text { if } M_{r} \bmod 3=0 \\ \frac{4}{27} \cdot \frac{M_{r}\left(M_{r}+\frac{1}{2}\right)^{2}}{\left(M_{r}-1\right)} \cdot \frac{1}{M a} & \text { if } M_{r} \bmod 3=1 \\ \frac{4}{27} \cdot \frac{M_{r}\left(M_{r}-\frac{1}{2}\right)^{2}\left(M_{r}+1\right)}{\left(M_{r}-1\right)^{2} M a} & \text { if } M_{r} \bmod 3=2 .\end{cases}
$$

\footnotetext{
${ }^{3}$ As 39 is symmetric with respect to $L_{r}=M_{r} / 2$, we obtain two values
} for $L_{r}^{\mathrm{opt}}$ that both minimize the MSE and are equally valid.

\section{Asymptotic Efficiency of 1-D ESPRIT-type algorithms with Spatial Smoothing}

Furthermore, the optimal value for $L_{r}^{\mathrm{opt}}$ from Subsection VI-B allows to analytically compute the asymptotic efficiency of the considered $R$-D ESPRIT-type and $R$-D NC ESPRITtype algorithms with spatial smoothing for a single source. To this end, we utilize the simplified single source expressions of the deterministic $R$-D Cramér-Rao bound (CRB) and $R$-D $\mathrm{NC} \mathrm{CRB}$ in [10] and [19], respectively. As both expressions are the same, we here only state the conventional case from [10].

For the case of an $M$-element $R$-D uniform sampling grid with an $M_{r}$-element ULA in the $r$-th mode and a single source $(d=1)$, the deterministic $R$-D CRB can be simplified to [10]

$$
\boldsymbol{C}=\operatorname{diag}\left\{\left[C^{(1)}, \ldots, C^{(R)}\right]^{\mathrm{T}}\right\},
$$

where $C^{(r)}=\frac{1}{\hat{\rho}} \cdot \frac{6}{M\left(M_{r}^{2}-1\right)}$. Using (39) and 46), the asymptotic efficiency $\eta^{(r)}\left(L_{r}^{\mathrm{opt}}\right)=\lim _{\hat{\rho} \rightarrow \infty} C^{(r)} / \mathrm{MSE}_{\mathrm{SS}}^{(r)}\left(L_{r}^{\mathrm{opt}}\right)$ of the spatially smoothed versions of $R$-D Standard and $R$ D Unitary ESPRIT as well as $R$-D NC Standard and $R$ D NC Unitary ESPRIT can be computed in closed-form for arbitrary dimensions $R$. As an example, the asymptotic efficiency $\eta\left(L^{\mathrm{opt}}\right)$ for $R=1$ is given by

$$
\eta\left(L^{\mathrm{opt}}\right) \approx \begin{cases}\frac{8}{9} \cdot \frac{M^{2}}{M^{2}-1} & \text { if } M \bmod 3=0 \\ \frac{8}{9} \cdot \frac{\left(M+\frac{1}{2}\right)^{2}}{M(M+1)} & \text { if } M \bmod 3=1 \\ \frac{8}{9} \cdot \frac{\left(M-\frac{1}{2}\right)^{2}}{M(M-1)} & \text { if } M \bmod 3=2 .\end{cases}
$$

It should be noted that $\eta$ is only a function of the array geometry, i.e., the number of sensors $M$. Moreover, it is straightforward to see that the asymptotic efficiency is larger when $M$ is a multiple of 3 . As one of the main results from [47), we observe that $\lim _{M \rightarrow \infty} \eta\left(L^{\mathrm{opt}}\right)=8 / 9$ for 1-D ESPRIT-type/NC ESPRIT-type algorithms with spatial smoothing. In contrast, it was shown in [10] and [19] that their counterparts without spatial smoothing become less efficient for increasing $M$, i.e., for $M \rightarrow \infty$, we have $\eta \rightarrow 0$. Consequently, spatial smoothing provides a significant gain for large $M$.

\section{Simulation Results}

In this section, we present two sets of simulation results to assess the behavior of the derived performance analysis of ESPRIT-type algorithms based on spatial smoothing and to illustrate the analytical expressions for the single source case.

\section{A. Performance Analysis}

We first compare the square root of the analytical MSE expressions ("ana") in (27), (31), and 33) to the root mean square error (RMSE) of the empirical estimation errors ("emp") of the spatially smoothed (SpSm) versions of $R$ D Standard ESPRIT (SE SpSm), $R$-D Unitary ESPRIT (UE

$$
c_{p}=\frac{1}{3} \cdot\left(\min \left\{L_{p}, M_{p}-L_{p}\right\}+1\right)\left(\min \left\{L_{p}, M_{p}-L_{p}\right\}\left(2 \cdot \min \left\{L_{p}, M_{p}-L_{p}\right\}-3 \cdot M_{p}-2\right)+6 \cdot M_{\mathrm{sub}_{p}} L_{p}\right)-M_{\mathrm{sub}_{p}} L_{p}
$$


SpSm) as well $R$-D NC Standard ESPRIT (NC SE SpSm) and $R$-D NC Unitary ESPRIT (NC UE SpSm). For all ESPRITtype algorithms, LS is used to solve the shift invariance equations. We also include the deterministic Cramér-Rao bounds for arbitrary signals (Det CRB) and strictly SO non-circular sources (Det NC CRB) [38]. The RMSE is defined as

$$
\mathrm{RMSE}=\sqrt{\frac{1}{R d} \mathbb{E}\left\{\sum_{r=1}^{R} \sum_{i=1}^{d}\left(\mu_{i}^{(r)}-\hat{\mu}_{i}^{(r)}\right)^{2}\right\}},
$$

where $\hat{\mu}_{i}^{(r)}$ is the estimate of $i$-th spatial frequency in the $r$-th mode. It is assumed that a known number of signals with unit power impinge on uniform array structures consisting of isotropic sensor elements with $\lambda / 2$-interelement spacing in all dimensions. The phase reference is located at the array centroid. The symbols $\boldsymbol{S}_{0}$ are drawn from a real-valued Gaussian distribution and we assume zero-mean circularly symmetric white Gaussian noise. The curves are averaged over 5000 Monte Carlo trials.

In Fig. 1, we depict the total RMSE versus the SNR of $d=2$ sources impinging on a $6 \times 6 \times 6$ uniform cubic array $(R=3)$ with $N=5$. The sources are located at $\mu_{1}^{(1)}=0$, $\mu_{2}^{(1)}=0.1, \mu_{1}^{(2)}=0, \mu_{2}^{(2)}=0.1, \mu_{1}^{(3)}=0$, and $\mu_{2}^{(3)}=0.1$. They have a pair-wise correlation of $\varrho=0.9$ and their rotation phases contained in $\Psi$ are given by $\varphi_{1}=0$ and $\varphi_{2}=\pi / 2$. For $L_{r}$, we choose $L_{r}^{\text {opt }}=M_{r} / 3=2$ in each mode, i.e., we have divided the array into a total of $L=8$ subarrays. Fig. 2 investigates the total RMSE versus the number of snapshots $N$ for a $6 \times 6$ uniform rectangular array (URA) $(R=2)$, where the SNR is $20 \mathrm{~dB}$ and $L_{r}=L_{r}^{\mathrm{opt}}=M_{r} / 3=3$. We have $d=3$ uncorrelated $(\varrho=0)$ sources at $\mu_{1}^{(1)}=0.25, \mu_{2}^{(1)}=0.5$, $\mu_{3}^{(1)}=0.75, \mu_{1}^{(2)}=0.25, \mu_{2}^{(2)}=0.5$, and $\mu_{3}^{(2)}=0.75$. The rotation phases are given by $\varphi_{1}=0, \varphi_{2}=\pi / 4$, and $\varphi_{3}=\pi / 2$.

It is apparent from Fig. 1 and Fig. 2 that the analytical results agree well with the empirical results for high effective SNRs, i.e., either high SNRs or a large sample size. Furthermore, NC SE SpSm and NC UE SpSm provide the lowest estimation errors and perform asymptotically identical at high effective SNRs. However, NC UE SpSm should be preferred due to its lower complexity and its better performance at low SNRs.

\section{B. Analytical Results for a Single Source}

In this subsection, the derived analytical results ("ana") in (44) and (47) for a single source $(d=1)$ are compared to their empirical versions. We also include the analytical and empirical single source results from [10] and [19] without spatial smoothing. The source is located at $\mu^{(r)}=0, \forall r$ (however, its location has no impact on the MSE) and the effective $\operatorname{SNR} \rho$ is $46 \mathrm{~dB}$ with $P=1, N=4$, and $\sigma_{\mathrm{n}}^{2}=10^{-4}$.

Fig. 3 illustrates the total RMSE using (44) as a function of the number of sensors $M_{1}=M_{2}$ for a 2-D $M_{1} \times M_{2}$ URA. We observe that the spatial smoothing based ESPRIT-type algorithms perform considerably closer to the CRB compared to the algorithms without spatial smoothing.

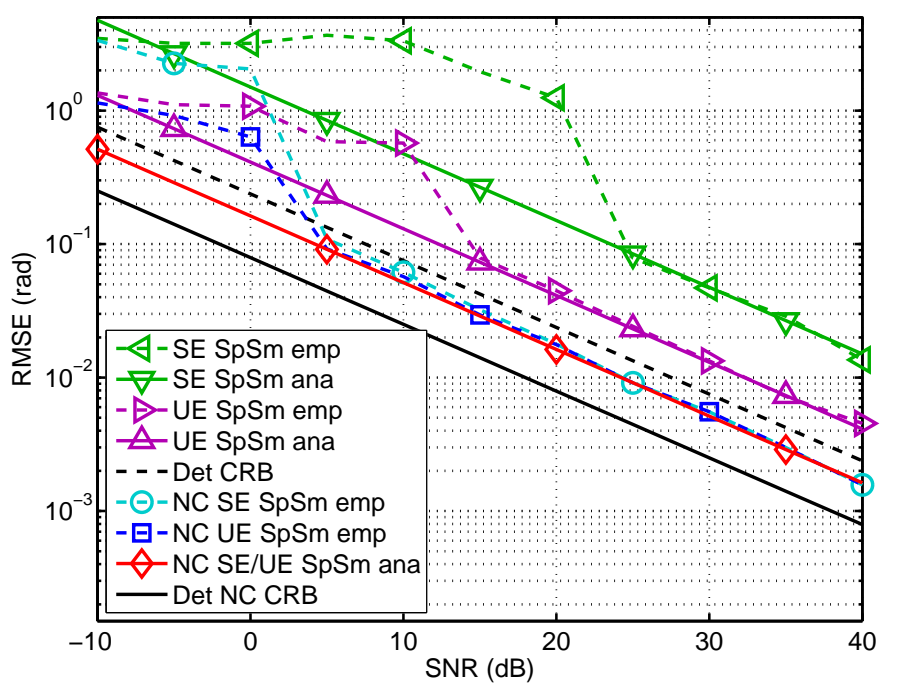

Fig. 1. RMSE versus SNR for a $6 \times 6 \times 6$ uniform cubic array $(R=3)$, and $N=5, d=2$ with $\varrho=0.9$ at $\mu_{1}^{(1)}=0, \mu_{2}^{(1)}=0.1, \mu_{1}^{(2)}=0$, $\mu_{2}^{(2)}=0.1, \mu_{1}^{(3)}=0, \mu_{2}^{(3)}=0.1$.

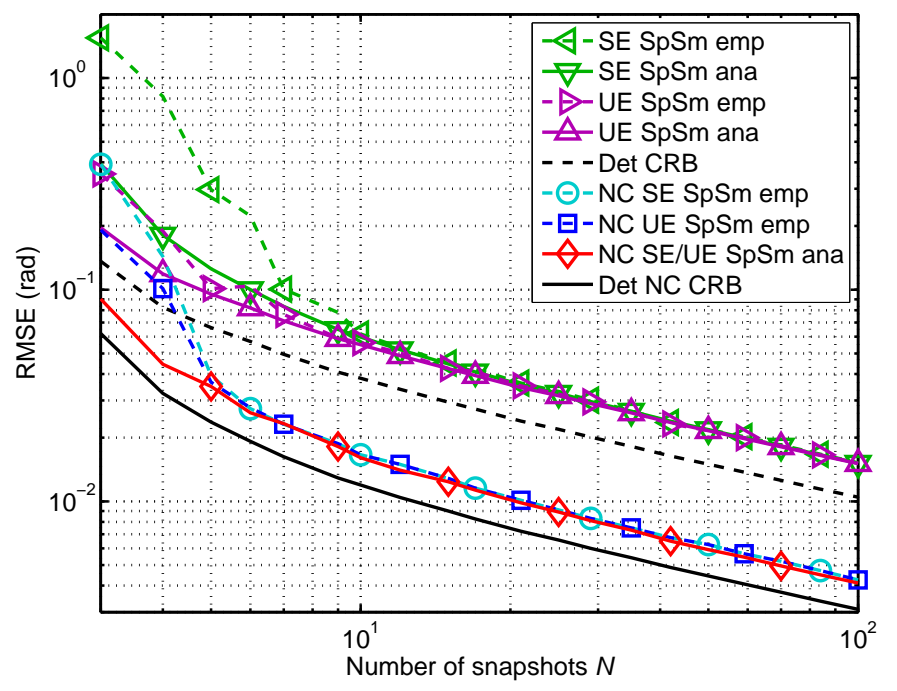

Fig. 2. RMSE versus $N$ for a $6 \times 6 \mathrm{URA}(R=2)$ and $\mathrm{SNR}=20 \mathrm{~dB}$, $d=3$ with $\varrho=0$ at $\mu_{1}^{(1)}=0.25, \mu_{2}^{(1)}=0.5, \mu_{3}^{(1)}=0.75, \mu_{1}^{(2)}=0.25$, $\mu_{2}^{(2)}=0.5, \mu_{3}^{(2)}=0.75$.

Fig. 4 presents the asymptotic efficiency (47) for $R=1$ versus $M$ of a ULA. The asymptotic efficiency for the non-spatial smoothing case, i.e., $L=1$, is given by $\eta(L=1)=\frac{6(M-1)}{M(M+1)}$. It is clear from Fig. 4 that all the algorithms are asymptotically efficient for $M=2$ and $M=3$. As $M$ increases further, the efficiency of the algorithms with spatial smoothing approaches the value $8 / 9$, while that of the non-spatial smoothing based algorithms becomes increasingly inefficient. Moreover, Fig. 4 confirms the observation from t47 that $\eta\left(L^{\mathrm{opt}}\right)$ is slightly higher for values of $M$ that are multiples of 3 .

\section{CONCLUSION}

This paper presents a first-order performance analysis of the spatially smoothed versions of $R$-D Standard ESPRIT and $R$-D Unitary ESPRIT for arbitrary sources as well as $R$ D NC Standard ESPRIT and $R$-D NC Unitary ESPRIT for 


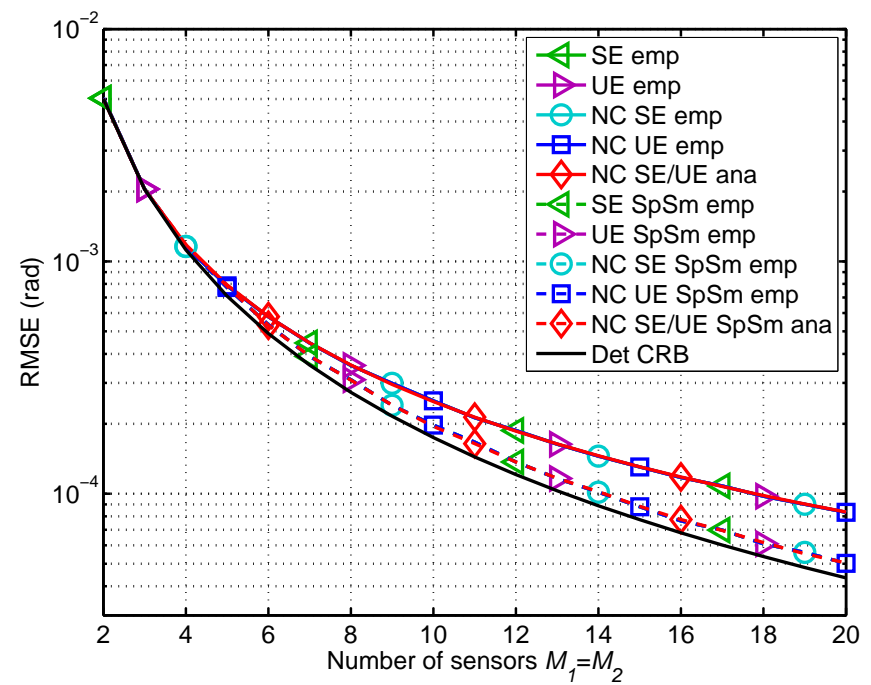

Fig. 3. RMSE versus $M_{1}=M_{2}$ of a $M_{1} \times M_{2}$ URA $(R=2)$ for $d=1$ at $\mu^{(1)}=0, \mu^{(2)}=0$, and $\rho=46 \mathrm{~dB}\left(P=1, N=4, \sigma_{n}^{2}=10^{-4}\right)$.

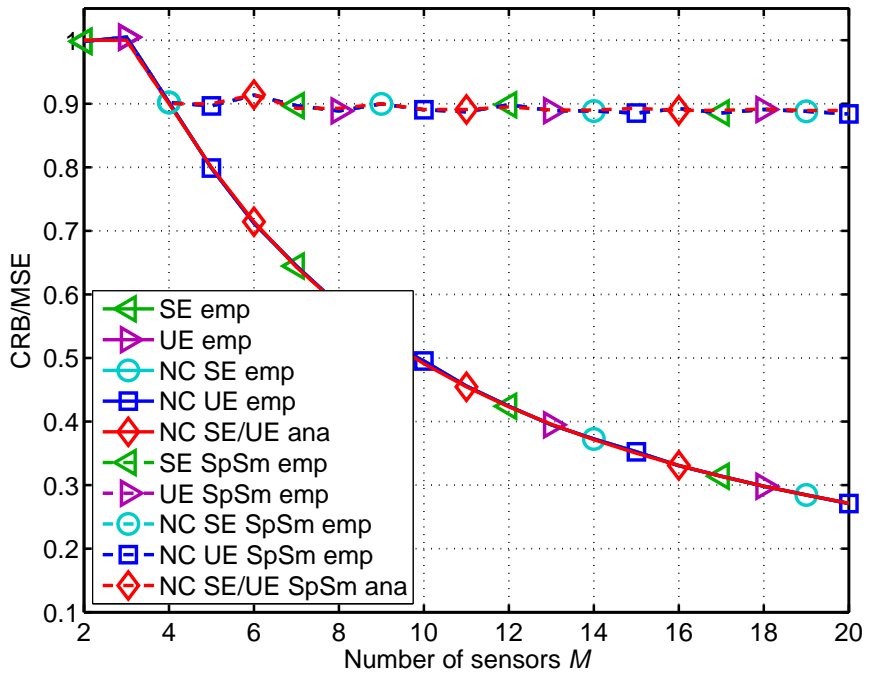

Fig. 4. Asymptotic efficiency versus $M$ of a ULA $(R=1)$ for $d=1$ at $\mu=0$ and $\rho=46 \mathrm{~dB}\left(P=1, N=4, \sigma_{n}^{2}=10^{-4}\right)$.

strictly SO non-circular sources. The derived expressions are asymptotic in the effective SNR and no assumptions on the noise statistics are required apart from a zero-mean and finite SO moments. We show that both spatially smoothed $R$-D NC ESPRIT-type algorithms perform asymptotically identical in the high effective SNR regime. As the performance generally depends on the number of subarrays, we have simplified the derived $R$-D MSE expressions for the special case of a single source, which allows to analytically compute the optimal number of subarrays for spatial smoothing. Additionally, we have derived the asymptotic spatial smoothing gain and calculated the asymptotic efficiency for this special case. The analytical results are supported by simulations.

\section{APPENDIX A}

PROOF OF THEOREM 1

To show this result, we simply use the FBA-processed and spatially smoothed augmented measurement matrix

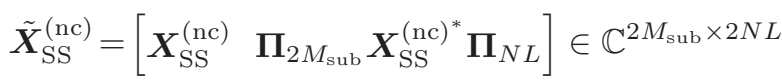

and compute the Gram matrix $\boldsymbol{G}=\tilde{\boldsymbol{X}}_{\mathrm{SS}}^{(\mathrm{nc})} \tilde{\boldsymbol{X}}_{\mathrm{SS}}^{(\mathrm{nc})^{\mathrm{H}}}$, which yields

$$
\boldsymbol{G}=\boldsymbol{X}_{\mathrm{SS}}^{(\mathrm{nc})} \boldsymbol{X}_{\mathrm{SS}}^{(\mathrm{nc})^{\mathrm{H}}}+\boldsymbol{\Pi}_{2 M_{\mathrm{sub}}} \boldsymbol{X}_{\mathrm{SS}}^{(\mathrm{nc})^{*}} \boldsymbol{X}_{\mathrm{SS}}^{(\mathrm{nc})^{\mathrm{T}}} \boldsymbol{\Pi}_{2 M_{\mathrm{sub}}} .
$$

Expanding the second term of (50) using (21), we obtain

$$
\begin{aligned}
& \boldsymbol{\Pi}_{2 M_{\mathrm{sub}}}\left(\sum_{\underline{\underline{\ell}}=\underline{\mathbf{1}}}^{\underline{\boldsymbol{L}}} \boldsymbol{J}_{\underline{\boldsymbol{\ell}}}^{(\mathrm{nc})} \boldsymbol{X}^{(\mathrm{nc})^{*}} \boldsymbol{X}^{(\mathrm{nc})^{\mathrm{T}}} \boldsymbol{J}_{\underline{\boldsymbol{\ell}}}^{(\mathrm{nc})^{\mathrm{T}}}\right) \boldsymbol{\Pi}_{2 M_{\mathrm{sub}}}
\end{aligned}
$$

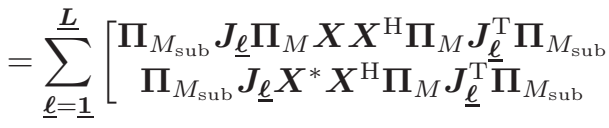

$$
\begin{aligned}
& \left.\begin{array}{c}
\boldsymbol{\Pi}_{M_{\mathrm{sub}}} \boldsymbol{J}_{\underline{\ell}} \boldsymbol{\Pi}_{M} \boldsymbol{X} \boldsymbol{X}^{\mathrm{T}} \boldsymbol{J}_{\underline{\ell}}^{\mathrm{T}} \boldsymbol{\Pi}_{M_{\mathrm{sub}}} \\
\boldsymbol{\Pi}_{M_{\mathrm{sub}}} \boldsymbol{J}_{\underline{\ell}} \boldsymbol{X}^{*} \boldsymbol{X}^{\mathrm{T}} \boldsymbol{J}_{\underline{\ell}}^{\mathrm{T}} \underline{\boldsymbol{\Pi}}_{M_{\mathrm{sub}}}
\end{array}\right] .
\end{aligned}
$$

Next, we observe the symmetries $\Pi_{M_{\mathrm{sub}}} \boldsymbol{J}_{\underline{\ell}} \boldsymbol{\Pi}_{M}=\boldsymbol{J}_{\underline{L}-\underline{\ell}+\underline{1}}$ and $\Pi_{M_{\mathrm{sub}}} \boldsymbol{J}_{\underline{\ell}}=\boldsymbol{J}_{\underline{\boldsymbol{L}}-\underline{\ell}+\underline{\mathbf{1}}} \boldsymbol{\Pi}_{M}$. Hence, we perform a change of variables to $\underline{m}=\underline{L}-\underline{\ell}+\underline{1}$, which simplifies (51) to

$$
\begin{aligned}
& \sum_{\underline{\boldsymbol{m}}=\underline{\boldsymbol{1}}}^{\underline{\boldsymbol{L}}}\left[\begin{array}{cc}
\boldsymbol{J}_{\underline{\boldsymbol{m}}} \boldsymbol{X} \boldsymbol{X}^{\mathrm{H}} \boldsymbol{J}_{\underline{\boldsymbol{m}}}^{\mathrm{T}} & \boldsymbol{J}_{\underline{\boldsymbol{m}}} \boldsymbol{X} \boldsymbol{X}^{\mathrm{T}} \boldsymbol{\Pi}_{M} \boldsymbol{J}_{\underline{\boldsymbol{m}}}^{\mathrm{T}} \\
\boldsymbol{J}_{M} \boldsymbol{X}^{*} \boldsymbol{X}^{\underline{\mathrm{H}} \boldsymbol{J}_{\underline{\boldsymbol{m}}}^{\mathrm{T}}} & \boldsymbol{J}_{\underline{\boldsymbol{m}}} \underline{\boldsymbol{\Pi}}_{M} \boldsymbol{X}^{*} \boldsymbol{X}^{\mathrm{T}} \boldsymbol{\Pi}_{M} \boldsymbol{J}_{\underline{\boldsymbol{m}}}^{\mathrm{T}}
\end{array}\right] \\
& =\boldsymbol{X}_{\mathrm{SS}}^{(\mathrm{nc})} \boldsymbol{X}_{\mathrm{SS}}^{(\mathrm{nc})^{\mathrm{H}}} .
\end{aligned}
$$

Replacing the second term of (50) by (52), we have $G=$ $2 \cdot \boldsymbol{X}_{\mathrm{SS}}^{(\mathrm{nc})} \boldsymbol{X}_{\mathrm{SS}}^{(\mathrm{nc})^{\mathrm{H}}}$. Thus, the matrix $\boldsymbol{G}$ reduces to the scaled Gram matrix of $\boldsymbol{X}_{\mathrm{SS}}^{(\mathrm{nc})}$, i.e., the column space of $\boldsymbol{X}_{\mathrm{SS}}^{(\mathrm{nc})}$ is the same as the column space of the Gram matrix of $\boldsymbol{X}_{\mathrm{SS}}^{(\mathrm{nc})}$. Consequently, FBA has no effect on the column space of $\boldsymbol{X}_{\mathrm{SS}}^{(\mathrm{nc})}$. This completes the proof.

\section{APPENDIX B}

\section{PROOF OF THEOREM 3}

This theorem consists of several parts, which we address in separate subsections.

\section{A. MSE for R-D Standard ESPRIT with Spatial Smoothing}

We start the proof by simplifying the MSE expression for $R$-D Standard ESPRIT with spatial smoothing in (27) and for $d=1$. In the single source case the noise-free spatially smoothed measurement matrix $\boldsymbol{X}_{\mathrm{SS}_{0}} \in \mathbb{C}^{M_{\mathrm{sub}} \times N L}$ can be written as

$$
\begin{aligned}
\boldsymbol{X}_{\mathrm{SS}_{0}} & =\overline{\boldsymbol{a}}_{\mathrm{SS}}(\boldsymbol{\mu}) \boldsymbol{\phi}^{\mathrm{T}}\left(\boldsymbol{I}_{L} \otimes \overline{\boldsymbol{s}}^{\mathrm{T}}\right)=\overline{\boldsymbol{a}}_{\mathrm{SS}}(\boldsymbol{\mu}) \boldsymbol{a}_{L}^{\mathrm{T}}\left(\boldsymbol{I}_{L} \otimes \overline{\boldsymbol{s}}^{\mathrm{T}}\right) \\
& =\overline{\boldsymbol{a}}_{\mathrm{SS}}(\boldsymbol{\mu})\left(\boldsymbol{a}_{L} \otimes \overline{\boldsymbol{s}}\right)^{\mathrm{T}}=\overline{\boldsymbol{a}}_{\mathrm{SS}}(\boldsymbol{\mu}) \overline{\boldsymbol{s}}_{L}^{\mathrm{T}}
\end{aligned}
$$

where $\overline{\boldsymbol{a}}_{\mathrm{SS}}(\boldsymbol{\mu})=\overline{\boldsymbol{a}}_{1}^{(1)}\left(\mu^{(1)}\right) \otimes \cdots \otimes \overline{\boldsymbol{a}}_{1}^{(R)}\left(\mu^{(R)}\right) \in \mathbb{C}^{M_{\mathrm{sub} \times 1}}$ is the spatially smoothed array steering vector in all $R$ modes with $\overline{\boldsymbol{a}}_{1}^{(r)}\left(\mu^{(r)}\right)=\boldsymbol{J}_{1_{r}}^{\left(M_{r}\right)} \overline{\boldsymbol{a}}^{(r)}\left(\mu^{(r)}\right) \in \mathbb{C}^{M_{\mathrm{sub}_{r}} \times 1}, \quad r=$ $1, \ldots, R$ and $\boldsymbol{\phi}=\boldsymbol{a}_{L}=\boldsymbol{a}_{L_{1}}^{(1)}\left(\mu^{(1)}\right) \otimes \cdots \otimes \boldsymbol{a}_{L_{R}}^{(R)}\left(\mu^{(R)}\right) \in \mathbb{C}^{L \times 1}$ with $\boldsymbol{a}_{L_{r}}^{(r)}\left(\mu^{(r)}\right)=\left[1, \mathrm{e}^{\mathrm{j} \mu^{(r)}}, \ldots, \mathrm{e}^{\mathrm{j} \mu^{(r)}\left(L_{r}-1\right)}\right]^{\mathrm{T}} \in \mathbb{C}^{L_{r} \times 1}, \forall r$. 
Moreover, $\bar{s} \in \mathbb{C}^{N \times 1}$ contains the source symbols with the empirical source power $\hat{P}_{\mathrm{s}}=\|\overline{\boldsymbol{s}}\|_{2}^{2} / N$ and we have $\overline{\boldsymbol{s}}_{L}^{\mathrm{H}} \overline{\boldsymbol{s}}_{L}=N L \hat{P}_{\mathrm{S}}$. In what follows, we drop the dependence of $\overline{\boldsymbol{a}}_{\mathrm{SS}}(\boldsymbol{\mu})$ on $\boldsymbol{\mu}$ for notational convenience. For a ULA of isotropic elements in each of the $R$ modes, $\overline{\boldsymbol{a}}^{(r)}$ is given by (6) and $\left\|\overline{\boldsymbol{a}}_{\mathrm{SS}}\right\|_{2}^{2}=M_{\mathrm{sub}}=M-L+1$. The selection matrices $\tilde{\boldsymbol{J}}_{\mathrm{SS}_{1}}^{(r)}$

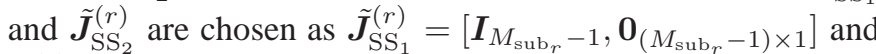
$\tilde{\boldsymbol{J}}_{\mathrm{SS}_{2}}^{(r)}=\left[\mathbf{0}_{\left(M_{\mathrm{sub}_{r}}-1\right) \times 1}, \boldsymbol{I}_{M_{\mathrm{sub}_{r}-1}}\right]$ for maximum overlap, i.e., $M_{\mathrm{sub}_{r}}^{(\mathrm{sel})}=M_{\mathrm{sub}_{r}}-1$. Note that (53) is a rank-one matrix and we can directly determine the subspaces from the SVD as

$$
\begin{aligned}
\boldsymbol{U}_{\mathrm{SS}_{\mathrm{s}}} & =\boldsymbol{u}_{\mathrm{SS}_{\mathrm{s}}}=\frac{\overline{\boldsymbol{a}}_{\mathrm{SS}}}{\| \overline{\boldsymbol{a}}_{\mathrm{SS} \|_{2}}}=\frac{\overline{\boldsymbol{a}}_{\mathrm{SS}}}{\sqrt{M_{\mathrm{sub}}}} \\
\boldsymbol{\Sigma}_{\mathrm{SS}_{\mathrm{s}}} & =\sigma_{\mathrm{SS}_{\mathrm{s}}}=\sqrt{M_{\mathrm{sub}} N L \hat{P}_{\mathrm{s}}} \\
\boldsymbol{V}_{\mathrm{SS}_{\mathrm{s}}} & =\boldsymbol{v}_{\mathrm{SS}_{\mathrm{s}}}=\frac{\overline{\boldsymbol{s}}_{L}^{*}}{\left\|\overline{\boldsymbol{s}}_{L}\right\|_{2}}=\frac{\overline{\boldsymbol{s}}_{L}^{*}}{\sqrt{N L \hat{P}_{\mathrm{s}}}} .
\end{aligned}
$$

For the MSE expression in (27), we also require $\boldsymbol{P}_{\bar{a}_{S S}}^{\perp}=$ $\boldsymbol{U}_{\mathrm{SS}_{\mathrm{n}}} \boldsymbol{U}_{\mathrm{SS}}^{\mathrm{H}}=\boldsymbol{I}_{M_{\mathrm{sub}}}-\frac{1}{M_{\mathrm{sub}}} \overline{\boldsymbol{a}}_{\mathrm{SS}} \overline{\boldsymbol{a}}_{\mathrm{SS}}^{\mathrm{H}}$, which is the projection matrix onto the noise subspace. Moreover, we have $\boldsymbol{\Phi}^{(r)}=$ $\mathrm{e}^{\mathrm{j} \mu^{(r)}}$ and hence, the eigenvectors are $\boldsymbol{p}_{i}^{(r)}=\boldsymbol{q}_{i}^{(r)}=1$. The SO moments $\boldsymbol{R}_{\mathrm{SS}}$ and $\boldsymbol{C}_{\mathrm{SS}}$ of the noise are given by (29) with $\boldsymbol{R}_{\mathrm{nn}}=\sigma_{\mathrm{n}}^{2} \boldsymbol{I}_{M}$ and $\boldsymbol{C}_{\mathrm{nn}}=\mathbf{0}$.

Inserting these expressions into 27, we get

$$
\mathbb{E}\left\{\left(\Delta \mu^{(r)}\right)^{2}\right\}=\frac{1}{2} \cdot \boldsymbol{z}^{\mathrm{H}} \boldsymbol{R}_{\mathrm{SS}}^{\mathrm{T}} \boldsymbol{z}=\frac{1}{2} \cdot \boldsymbol{z}^{\mathrm{T}} \boldsymbol{R}_{\mathrm{SS}} \boldsymbol{z}^{*}
$$

with $\boldsymbol{z}=\boldsymbol{W}_{\mathrm{SS}^{\mathrm{T}}}^{\mathrm{T}} \boldsymbol{r}_{\mathrm{SS}_{i}}^{(r)}$ and

$$
\begin{aligned}
\boldsymbol{r}_{\mathrm{SS}_{i}}^{(r)} & =\left[\left(\tilde{\boldsymbol{J}}_{\mathrm{SS}_{1}}^{(r)} \frac{\overline{\boldsymbol{a}}_{\mathrm{SS}}}{\sqrt{M_{\mathrm{sub}}}}\right)^{+}\left(\tilde{\boldsymbol{J}}_{\mathrm{SS}_{2}}^{(r)} / \mathrm{e}^{\mathrm{j} \mu^{(r)}}-\tilde{\boldsymbol{J}}_{\mathrm{SS}_{1}}^{(r)}\right)\right]^{\mathrm{T}} \in \mathbb{C}^{M_{\mathrm{sub}} \times 1}, \\
\boldsymbol{W}_{\mathrm{SS}} & =\left(\frac{1}{\sqrt{M_{\mathrm{sub}} N L \hat{P}_{\mathrm{S}}}} \cdot \frac{\overline{\boldsymbol{s}}_{L}^{\mathrm{H}}}{\sqrt{N L \hat{P}_{\mathrm{S}}}}\right) \otimes \boldsymbol{P}_{\overline{\boldsymbol{a}}_{\mathrm{SS}}}^{\perp} \in \mathbb{C}^{M_{\mathrm{sub}} \times M_{\mathrm{sub}} N L} .
\end{aligned}
$$

Note that the term $\boldsymbol{z}^{\mathrm{T}}$ can also be written as $\boldsymbol{z}^{\mathrm{T}}=\tilde{\boldsymbol{s}}^{\mathrm{T}} \otimes \tilde{\boldsymbol{a}}^{(r)^{\mathrm{T}}}$, where

$$
\begin{aligned}
& \tilde{\boldsymbol{s}}^{\mathrm{T}}=\frac{1}{\sqrt{M_{\mathrm{sub}} N L \hat{P}_{\mathrm{S}}}} \cdot \frac{\left(\boldsymbol{a}_{L} \otimes \overline{\boldsymbol{s}}\right)^{\mathrm{H}}}{\sqrt{N L \hat{P}_{\mathrm{s}}}} \\
& \tilde{\boldsymbol{a}}^{(r)^{\mathrm{T}}}=\left(\tilde{\boldsymbol{J}}_{\mathrm{SS}_{1}}^{(r)} \frac{\overline{\boldsymbol{a}}_{\mathrm{SS}}}{\sqrt{M_{\mathrm{Sub}}}}\right)^{+}\left(\tilde{\boldsymbol{J}}_{\mathrm{SS}_{2}}^{(r)} / \mathrm{e}^{\mathrm{j} \mu(r)}-\tilde{\boldsymbol{J}}_{\mathrm{SS}_{1}}^{(r)}\right) \boldsymbol{P}_{\overline{\boldsymbol{a}}_{\mathrm{SS}}}^{\perp} .
\end{aligned}
$$

Next, we further simplify the expression $\tilde{\boldsymbol{a}}^{(r)^{\mathrm{T}}}$ and expand the pseudo-inverse of $\tilde{\boldsymbol{J}}_{\mathrm{SS}_{1}}^{(r)} \overline{\boldsymbol{a}}_{\mathrm{SS}}$ using the relation $\boldsymbol{x}^{+}=\boldsymbol{x}^{\mathrm{H}} /\|\boldsymbol{x}\|_{2}^{2}$. As $\tilde{\boldsymbol{J}}_{\mathrm{SS}_{1}}^{(r)}$ selects $M_{\mathrm{sub}_{r}}-1$ out of $M_{\mathrm{sub}_{r}}$ elements in the $r$-th mode, we have $\left\|\tilde{\boldsymbol{J}}_{\mathrm{SS}_{1}}^{(r)} \overline{\boldsymbol{a}}_{\mathrm{SS}}\right\|_{2}^{2}=\frac{M_{\mathrm{sub}}}{M_{\mathrm{sub}_{r}}} \cdot\left(M_{\mathrm{Sub}_{r}}-1\right)$. Then, taking the shift invariance equation $\tilde{\boldsymbol{J}}_{\mathrm{SS}_{2}}^{(r)} \overline{\boldsymbol{a}}_{\mathrm{SS}} / \mathrm{e}^{\mathrm{j} \mu^{(r)}}-$ $\tilde{\boldsymbol{J}}_{\mathrm{SS}_{1}}^{(r)} \overline{\boldsymbol{a}}_{\mathrm{SS}}=\mathbf{0}$ in the $r$-th mode into account, we obtain

$$
\begin{aligned}
\tilde{\boldsymbol{a}}^{(r)^{\mathrm{T}}} & =\frac{\sqrt{M_{\mathrm{Sub}}} M_{\mathrm{Sub}_{r}}}{M_{\mathrm{Sub}}\left(M_{\mathrm{Sub}_{r}}-1\right)} \cdot \check{\boldsymbol{a}}^{(r)^{\mathrm{T}}}, \\
\check{\boldsymbol{a}}^{(r)^{\mathrm{T}}} & =\overline{\boldsymbol{a}}_{\mathrm{SS}}^{\mathrm{H}}\left(\tilde{\boldsymbol{J}}_{\mathrm{SS}_{2}}^{(r)} \tilde{\boldsymbol{J}}_{\mathrm{SS}_{2}}^{\mathrm{H}}-\tilde{\boldsymbol{J}}_{\mathrm{SS}_{1}}^{(r)^{\mathrm{H}}} \tilde{\boldsymbol{J}}_{\mathrm{SS}_{1}}^{(r)}\right) .
\end{aligned}
$$

Since the vector $\overline{\boldsymbol{a}}_{\mathrm{SS}}$ and the matrices $\tilde{\boldsymbol{J}}_{\mathrm{SS}}^{(r)}, \quad k=1,2$, contained in $\overline{\boldsymbol{a}}^{(r)^{\mathrm{T}}}$ can be written as $\overline{\boldsymbol{a}}_{\mathrm{SS}}=\overline{\boldsymbol{a}}_{1}^{(1)} \otimes \cdots \otimes \overline{\boldsymbol{a}}_{1}^{(R)}$

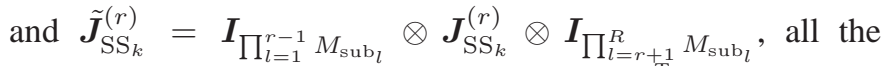
unaffected modes can be factored out of $\check{\boldsymbol{a}}^{(r)^{\mathrm{T}}}$, yielding

$$
\begin{aligned}
\check{\boldsymbol{a}}^{(r)^{\mathrm{T}}}= & \left(\overline{\boldsymbol{a}}_{1}^{(1)} \otimes \cdots \otimes \overline{\boldsymbol{a}}_{1}^{(r-1)}\right)^{\mathrm{H}} \otimes \breve{\boldsymbol{a}}_{1}^{(r)^{\mathrm{T}}} \\
& \otimes\left(\overline{\boldsymbol{a}}_{1}^{(r+1)} \otimes \cdots \otimes \overline{\boldsymbol{a}}_{1}^{(R)}\right)^{\mathrm{H}},
\end{aligned}
$$

where we have $\breve{\boldsymbol{a}}_{1}^{(r)^{\mathrm{T}}}=\overline{\boldsymbol{a}}_{1}^{(r)^{\mathrm{H}}}\left(\boldsymbol{J}_{\mathrm{SS}_{2}}^{(r)^{\mathrm{H}}} \boldsymbol{J}_{\mathrm{SS}_{2}}^{(r)}-\boldsymbol{J}_{\mathrm{SS}_{1}}^{(r)^{\mathrm{H}}} \boldsymbol{J}_{\mathrm{SS}_{1}}^{(r)}\right)$ with $\overline{\boldsymbol{a}}_{1}^{(r)^{\mathrm{H}}}=\left[\mathrm{e}^{\mathrm{j} \frac{\left(M_{r}-1\right)}{2} \mu^{(r)}}, \ldots, \mathrm{e}^{-\mathrm{j} \frac{\left(M_{r}-2 L_{r}-1\right)}{2} \mu^{(r)}}, \mathrm{e}^{-\mathrm{j} \frac{\left(M_{r}-2 L_{r}+1\right)}{2} \mu^{(r)}}\right]$. Then, it is easy to verify that

$$
\breve{\boldsymbol{a}}_{1}^{(r)^{\mathrm{T}}}=\left[-\mathrm{e}^{\mathrm{j} \frac{\left(M_{r}-1\right)}{2} \mu^{(r)}}, 0, \ldots, 0, \mathrm{e}^{-\mathrm{j} \frac{\left(M_{r}-2 L_{r}+1\right)}{2} \mu^{(r)}}\right] .
$$

Thus, the MSE expression in (54) is given by

$$
\mathbb{E}\left\{\left(\Delta \mu^{(r)}\right)^{2}\right\}=\frac{k^{2}}{2} \cdot \boldsymbol{v}^{\mathrm{T}} \boldsymbol{R}_{\mathrm{SS}} \boldsymbol{v}^{*}
$$

where we haved used $\boldsymbol{z}^{\mathrm{T}}=k \cdot \boldsymbol{v}^{\mathrm{T}}$ with $\boldsymbol{v}^{\mathrm{T}}=\boldsymbol{a}_{L}^{\mathrm{H}} \otimes \boldsymbol{s}^{\mathrm{H}} \otimes$ $\check{\boldsymbol{a}}^{(r)^{\mathrm{T}}}$ and $k=\frac{1}{N L \hat{P}_{\mathrm{s}}} \cdot \frac{M_{\mathrm{sub}_{r}}}{M_{\mathrm{sub}}\left(M_{\left.\mathrm{sub}_{r}-1\right)}\right.}$. After straightforward calculations, we further simplify 600 tobain (61) at the bottom of this page, where $c_{p}$ in 63 is given by 40 and it can be shown that the last term in (62) evaluates to $2 \cdot\left(L_{r}-\max \left\{2 \cdot L_{r}-M_{r}, 0\right\}\right)=2 \cdot \min \left\{L_{r}, M_{r}-L_{r}\right\}$. Consequently, the MSE of $R$-D Standard ESPRIT with spatial smoothing is given by

$$
\begin{aligned}
& \mathbb{E}\left\{\left(\Delta \mu^{(r)}\right)^{2}\right\}=\frac{\sigma_{\mathrm{n}}^{2}}{N \hat{P}_{\mathrm{s}}} \cdot \frac{M_{\mathrm{sub}_{r}}^{2} \min \left\{L_{r}, M_{r}-L_{r}\right\}}{L^{2} M_{\mathrm{sub}}^{2}\left(M_{\mathrm{sub}_{r}}-1\right)^{2}} \cdot \prod_{\substack{p=1 \\
p \neq r}}^{R} c_{p} \\
& =\frac{\sigma_{\mathrm{n}}^{2}}{N \hat{P}_{\mathrm{s}}} \cdot \frac{\min \left\{L_{r}, M_{r}-L_{r}\right\}}{\left(M_{r}-L_{r}\right)^{2} L_{r}^{2}} \cdot \prod_{\substack{p=1 \\
p \neq r}}^{R} \frac{c_{p}}{M_{\mathrm{sub}_{p}}^{2} L_{p}^{2}}
\end{aligned}
$$

where we have used the fact that $M_{\text {sub }}=M_{\mathrm{sub}_{r}} \cdot \prod_{\substack{p=1 \\ p \neq r}}^{R} M_{\mathrm{sub}_{p}}$ and $L=L_{r} \cdot \prod_{\substack{p=1 \\ p \neq r}}^{R} L_{p}$. Equation (64) is the desired result.

\section{B. MSE for R-D Unitary ESPRIT with Spatial Smoothing}

The second part of the theorem is to show that for a single source, the MSE of $R$-D Unitary ESPRIT with spatial smoothing in (31) is the same as the MSE of $R$-D Standard ESPRIT with spatial smoothing in (27). Firstly, we simplify $\tilde{\boldsymbol{X}}_{\mathrm{SS}_{0}}$ from 30 and find

$$
\begin{aligned}
\tilde{\boldsymbol{X}}_{\mathrm{SS}_{0}} & =\left[\begin{array}{ll}
\overline{\boldsymbol{a}}_{\mathrm{SS}} \overline{\boldsymbol{s}}_{L}^{\mathrm{T}} & \boldsymbol{\Pi}_{M_{\mathrm{sub}}} \overline{\boldsymbol{a}}_{\mathrm{SS}}^{*} \overline{\boldsymbol{s}}_{L}^{\mathrm{H}} \boldsymbol{\Pi}_{N L}
\end{array}\right] \\
& =\overline{\boldsymbol{a}}_{\mathrm{SS}}\left[\begin{array}{ll}
\overline{\boldsymbol{s}}_{L}^{\mathrm{T}} & \mathrm{e}^{\mathrm{j} \sum_{r=1}^{R}\left(L_{r}-1\right) \mu^{(r)}} \overline{\boldsymbol{s}}_{L}^{\mathrm{H}} \boldsymbol{\Pi}_{N L}
\end{array}\right] \\
& =\overline{\boldsymbol{a}}_{\mathrm{SS}} \overline{\overline{\boldsymbol{s}}}_{L}^{\mathrm{T}},
\end{aligned}
$$

where in 65, we have used the fact that $\Pi_{M_{\mathrm{sub}_{r}}} \overline{\boldsymbol{a}}_{1}^{(r)^{*}}\left(\mu^{(r)}\right)=$ $\overline{\boldsymbol{a}}_{1}^{(r)}\left(\mu^{(r)}\right) \mathrm{e}^{\mathrm{j}\left(L_{r}-1\right) \mu^{(r)}}$ holds for a ULA in the $r$-th mode. Moreover, we have defined

$$
\overline{\overline{\boldsymbol{s}}}_{L}=\left[\begin{array}{c}
\overline{\boldsymbol{s}}_{L} \\
\mathrm{e}^{\mathrm{j} \sum_{r=1}^{R}\left(L_{r}-1\right) \mu^{(r)}} \boldsymbol{\Pi}_{N L} \overline{\boldsymbol{s}}_{L}^{*}
\end{array}\right]=\left[\begin{array}{c}
\boldsymbol{a}_{L} \otimes \overline{\boldsymbol{s}} \\
\boldsymbol{a}_{L} \otimes \boldsymbol{\Pi}_{N} \overline{\boldsymbol{s}}^{*}
\end{array}\right] .
$$


Note that $\left\|\overline{\overline{\boldsymbol{s}}}_{L}\right\|_{2}^{2}=2 N L \hat{P}_{\mathrm{S}}$. The subspaces from the SVD of $\tilde{\boldsymbol{X}}_{\mathrm{SS}_{0}}$ are obtained as

$$
\begin{aligned}
& \tilde{\boldsymbol{u}}_{\mathrm{SS}_{\mathrm{s}}}=\frac{\overline{\boldsymbol{a}}_{\mathrm{SS}}}{\sqrt{M_{\mathrm{sub}}}}=\boldsymbol{u}_{\mathrm{SS}_{\mathrm{s}}}, \quad \tilde{\sigma}_{\mathrm{SS}_{\mathrm{s}}}=\sqrt{2 M_{\mathrm{sub}} N L \hat{P}_{\mathrm{S}}} \\
& \tilde{\boldsymbol{v}}_{\mathrm{SS}_{\mathrm{s}}}=\frac{\overline{\overline{\boldsymbol{s}}}_{L}^{*}}{\sqrt{2 N L \hat{P}_{\mathrm{s}}}} .
\end{aligned}
$$

Compared to the previous subsection, it is apparent that FBA does not affect the column space $\boldsymbol{u}_{\mathrm{SS}_{\mathrm{s}}}$, such that $\tilde{\boldsymbol{U}}_{\mathrm{SS}_{\mathrm{n}}}=$ $\boldsymbol{U}_{\mathrm{SS}_{\mathrm{n}}}$ and thus $\tilde{\boldsymbol{P}}_{\overline{\boldsymbol{a}}_{\mathrm{SS}}}^{\perp}=\boldsymbol{P}_{\overline{\boldsymbol{a}}_{\mathrm{SS}}}^{\perp}$. However, FBA destroys the circular symmetry of the noise, resulting in an additional term in the MSE expression. Following the derivation for $R-\mathrm{D}$ Standard ESPRIT with spatial smoothing, it can be shown that $\tilde{\boldsymbol{z}}^{\mathrm{T}}=\tilde{\boldsymbol{r}}_{\mathrm{SS}_{i}}^{(r)^{\mathrm{T}}} \tilde{\boldsymbol{W}}_{\mathrm{SS}}=\tilde{\tilde{\boldsymbol{s}}}^{\mathrm{T}} \otimes \tilde{\boldsymbol{a}}^{(r)^{\mathrm{T}}}$, where

$$
\tilde{\tilde{\boldsymbol{s}}}^{\mathrm{T}}=\frac{1}{\sqrt{2 M_{\mathrm{sub}} N L \hat{P}_{\mathrm{s}}}} \cdot \frac{\overline{\overline{\boldsymbol{s}}}_{L}^{\mathrm{H}}}{\sqrt{2 N L \hat{P}_{\mathrm{s}}}}
$$

and $\tilde{\boldsymbol{a}}^{(r)^{\mathrm{T}}}$ is given as in (56). Thus, the MSE expression for $R$-D Unitary ESPRIT with spatial smoothing in 31 can be written as

$$
\mathbb{E}\left\{\left(\Delta \mu^{(r)}\right)^{2}\right\}=\frac{1}{2} \cdot\left(\tilde{\boldsymbol{z}}^{\mathrm{T}} \tilde{\boldsymbol{R}}_{\mathrm{SS}} \tilde{\boldsymbol{z}}^{*}-\operatorname{Re}\left\{\tilde{\boldsymbol{z}}^{\mathrm{T}} \tilde{\boldsymbol{C}}_{\mathrm{SS}} \tilde{\boldsymbol{z}}\right\}\right)
$$

where $\tilde{\boldsymbol{R}}_{\mathrm{SS}}=\left(\boldsymbol{I}_{2} \otimes \boldsymbol{R}_{\mathrm{SS}}\right)$ and $\tilde{\boldsymbol{C}}_{\mathrm{SS}}=\left(\boldsymbol{\Pi}_{2} \otimes \boldsymbol{\Pi}_{M_{\mathrm{sub}} N L} \boldsymbol{R}_{\mathrm{SS}}\right)$. Expanding 69), we have

$$
\begin{aligned}
& \mathbb{E}\left\{\left(\Delta \mu^{(r)}\right)^{2}\right\}=\frac{\tilde{k}^{2}}{2} \cdot\left(\boldsymbol{v}^{\mathrm{T}} \boldsymbol{R}_{\mathrm{SS}} \boldsymbol{v}^{*}+\overline{\boldsymbol{v}}^{\mathrm{T}} \boldsymbol{R}_{\mathrm{SS}} \overline{\boldsymbol{v}}^{*}\right. \\
& \left.-\operatorname{Re}\left\{\boldsymbol{v}^{\mathrm{T}} \boldsymbol{\Pi}_{M_{\mathrm{sub}} N L} \boldsymbol{R}_{\mathrm{SS}} \overline{\boldsymbol{v}}+\overline{\boldsymbol{v}}^{\mathrm{T}} \boldsymbol{\Pi}_{M_{\mathrm{sub}} N L} \boldsymbol{R}_{\mathrm{SS}} \boldsymbol{v}^{*}\right\}\right)
\end{aligned}
$$

where $\tilde{\boldsymbol{z}}^{\mathrm{T}}=\tilde{k} \cdot \tilde{\boldsymbol{v}}^{\mathrm{T}}$ with $\tilde{\boldsymbol{v}}^{\mathrm{T}}=\left[\boldsymbol{v}^{\mathrm{T}}, \overline{\boldsymbol{v}}^{\mathrm{T}}\right], \overline{\boldsymbol{v}}^{\mathrm{T}}=\boldsymbol{a}_{L}^{\mathrm{H}} \otimes \boldsymbol{s}^{\mathrm{T}} \boldsymbol{\Pi}_{N} \otimes$

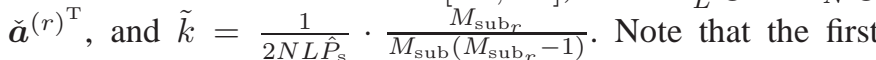
term of (70) was already computed in (63) as $2 \cdot \sigma_{\mathrm{n}}^{2} \cdot N \hat{P}_{\mathrm{s}}$. $\prod_{\substack{p=1 \\ p \neq r}}^{R} c_{p} \cdot \min \left\{L_{r}, M_{r}-L_{r}\right\}$. The remaining terms can be $p \neq r$
computed accordingly, where for the second term, we also obtain $2 \cdot \sigma_{\mathrm{n}}^{2} \cdot N \hat{P}_{\mathrm{s}} \cdot \prod_{\substack{p=1 \\ p \neq r}}^{R} c_{p} \cdot \min \left\{L_{r}, M_{r}-L_{r}\right\}$ while the third and fourth terms both evaluate to $-2 \cdot \sigma_{\mathrm{n}}^{2} \cdot N \hat{P}_{\mathrm{s}} \cdot \prod_{\substack{p=1 \\ p \neq r}}^{R} c_{p}$. $\min \left\{L_{r}, M_{r}-L_{r}\right\}$. Inserting these intermediate results into
(70), we obtain for the MSE of $R$-D Unitary ESPRIT with spatial smoothing

$$
\mathbb{E}\left\{\left(\Delta \mu^{(r)}\right)^{2}\right\}=\frac{\sigma_{\mathrm{n}}^{2}}{N \hat{P}_{\mathrm{s}}} \cdot \frac{\min \left\{L_{r}, M_{r}-L_{r}\right\}}{\left(M_{r}-L_{r}\right)^{2} L_{r}^{2}} \cdot \prod_{\substack{p=1 \\ p \neq r}}^{R} \frac{c_{p}}{M_{\operatorname{sub}_{p}}^{2} L_{p}^{2}},
$$

which is equal to 64 and hence proves this part.

\section{MSE for R-D NC Standard ESPRIT and Unitary ESPRIT with Spatial Smoothing}

The third part of the theorem is to show that the MSE of the spatially smoothed versions of $R$-D NC Standard ESPRIT and $R$-D NC Unitary ESPRIT is the same as the MSE for $R$-D Standard ESPRIT and Unitary ESPRIT. As we have already proven that the performance of $R$-D NC Standard and $R$-D NC Unitary ESPRIT with spatial smoothing is identical in the high effective SNR in general, this must also hold true for the case $d=1$. Hence, it is sufficient to simplify the MSE of $R$-D NC Standard ESPRIT in (33) for this special case.

We start by writing $\boldsymbol{X}_{\mathrm{SS}_{0}}^{(\mathrm{nc})}$ in (22) as

$$
\boldsymbol{X}_{\mathrm{SS}_{0}}=\overline{\boldsymbol{a}}_{\mathrm{SS}}^{\mathrm{nc})} \overline{\boldsymbol{s}}_{L}^{\mathrm{T}},
$$

where $\overline{\boldsymbol{s}}_{L}$ was defined in (53) and $\overline{\boldsymbol{a}}_{\mathrm{SS}}^{(\mathrm{nc})}=[1, \tilde{\Psi}]^{\mathrm{T}} \otimes \overline{\boldsymbol{a}}_{\mathrm{SS}}$ with $\tilde{\Psi}=\Psi^{*} \Psi^{*}=\mathrm{e}^{-\mathrm{j} 2 \varphi}$. This follows from 22 and the fact that $\overline{\boldsymbol{a}}^{(\mathrm{nc})}=[1, \tilde{\Psi}]^{\mathrm{T}} \otimes \overline{\boldsymbol{a}}$ for a uniform $R$-D array whose phase reference is at the centroid, i.e. $\boldsymbol{\Pi}_{M} \overline{\boldsymbol{a}}^{*}=\overline{\boldsymbol{a}}$ holds. Therefore, we have $\left\|\overline{\boldsymbol{a}}_{\mathrm{SS}}^{(\mathrm{nc})}\right\|_{2}^{2}=2 M_{\text {sub. }}$. The selection matrices $\tilde{\boldsymbol{J}}_{\mathrm{SS}_{k}}^{(\mathrm{nc})(r)}, k=1,2$ are given by $\tilde{\boldsymbol{J}}_{\mathrm{SS}_{k}}^{(\mathrm{nc})(r)}=\boldsymbol{I}_{2} \otimes \tilde{\boldsymbol{J}}_{\mathrm{SS}_{k}}^{(r)}$. The SVD of (72) can be explicitly expressed as

$$
\begin{aligned}
& \boldsymbol{u}_{\mathrm{SS}_{\mathrm{s}}}^{(\mathrm{nc})}=\frac{\overline{\boldsymbol{a}}_{\mathrm{SS}}^{(\mathrm{nc})}}{\sqrt{2 M_{\mathrm{sub}}}}, \quad \sigma_{\mathrm{SS}_{\mathrm{s}}}^{(\mathrm{nc})}=\sqrt{2 M_{\mathrm{sub}} N L \hat{P}_{\mathrm{s}}} \\
& \boldsymbol{v}_{\mathrm{SS}_{\mathrm{s}}}^{(\mathrm{nc})}=\frac{\overline{\boldsymbol{s}}_{L}^{*}}{\sqrt{N L \hat{P}_{\mathrm{s}}}}=\boldsymbol{v}_{\mathrm{SS}_{\mathrm{s}}} .
\end{aligned}
$$

It is evident that the $\mathrm{NC}$ preprocessing only affects the column space $\boldsymbol{u}_{\mathrm{SS}_{\mathrm{s}}}^{(\mathrm{nc})}$ while the row space $\boldsymbol{v}_{\mathrm{SS}_{\mathrm{s}}}$ of $R$-D Standard ESPRIT remains unaffected. Therefore, we have $\boldsymbol{P}_{\overline{\boldsymbol{a}}_{\mathrm{SS}}^{(\mathrm{nc})}}^{\perp}=$ $\boldsymbol{U}_{\mathrm{SS}_{\mathrm{n}}}^{(\mathrm{nc})} \boldsymbol{U}_{\mathrm{SS}_{\mathrm{n}}}^{(\mathrm{nc})^{\mathrm{H}}}=\boldsymbol{I}_{M_{\mathrm{sub}}}-\frac{1}{M_{\mathrm{sub}}} \overline{\boldsymbol{a}}_{\mathrm{SS}}^{(\mathrm{nc})} \overline{\boldsymbol{a}}_{\mathrm{SS}}^{(\mathrm{nc})^{\mathrm{H}}}$. Similarly to FBA,

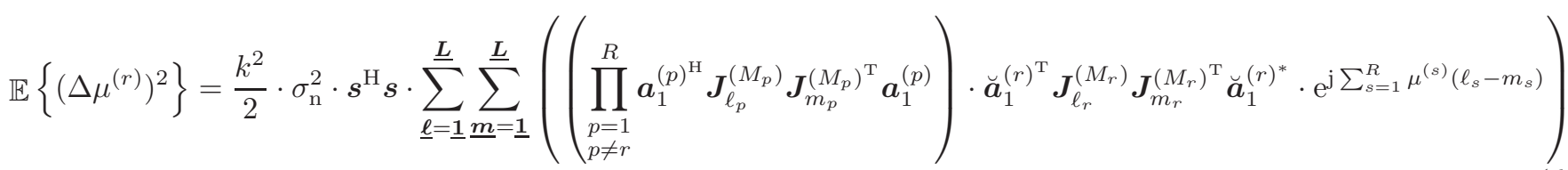

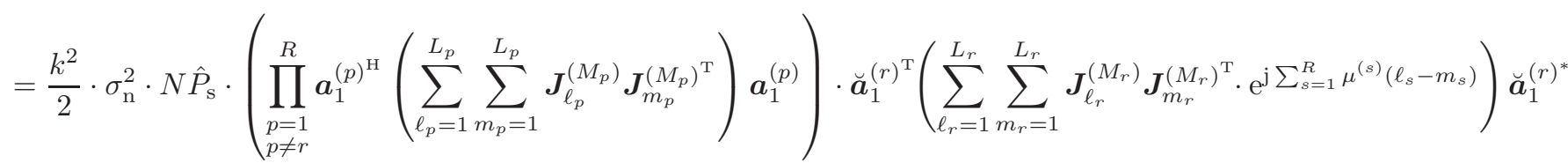

$=\frac{k^{2}}{2} \cdot \sigma_{\mathrm{n}}^{2} \cdot N \hat{P}_{\mathrm{s}} \cdot\left(\prod_{\substack{p=1 \\ p \neq r}}^{R} c_{p}\right) \cdot 2 \cdot \min \left\{L_{r}, M_{r}-L_{r}\right\}$ 
the circular symmetry of the noise is destroyed by the $\mathrm{NC}$ preprocessing step. In the $\mathrm{NC}$ case, it can be shown that $\boldsymbol{z}^{(\mathrm{nc})^{\mathrm{T}}}=\boldsymbol{r}_{\mathrm{SS}_{i}}^{(\mathrm{nc})(r)^{\mathrm{T}}} \boldsymbol{W}_{\mathrm{SS}}^{(\mathrm{nc})}=\tilde{\boldsymbol{s}}^{(\mathrm{nc})^{\mathrm{T}}} \otimes \tilde{\boldsymbol{a}}^{(\mathrm{nc})(r)^{\mathrm{T}}}$, where

$$
\begin{aligned}
\tilde{\boldsymbol{s}}^{(\mathrm{nc})^{\mathrm{T}}} & =\frac{1}{\sqrt{2 M_{\mathrm{sub}} N L \hat{P}_{\mathrm{S}}}} \cdot \frac{\left(\boldsymbol{a}_{L} \otimes \overline{\boldsymbol{s}}\right)^{\mathrm{H}}}{\sqrt{N L \hat{P}_{\mathrm{S}}}} \\
\tilde{\boldsymbol{a}}^{(\mathrm{nc})(r)^{\mathrm{T}}} & =\left(\tilde{\boldsymbol{J}}_{\mathrm{SS}_{1}(\mathrm{nc})(r)} \frac{\overline{\boldsymbol{a}}_{\mathrm{SS}}^{(\mathrm{nc})}}{\sqrt{2 M_{\mathrm{sub}}}}\right)^{+}\left(\tilde{\boldsymbol{J}}_{\mathrm{SS}_{2}}^{(\mathrm{nc})(r)} / \mathrm{e}^{\mathrm{j} \mu^{(r)}}-\tilde{\boldsymbol{J}}_{\mathrm{SS}_{1}}^{(\mathrm{nc})(r)}\right) \boldsymbol{P}_{\overline{\boldsymbol{a}}_{\mathrm{SS}}^{(\mathrm{nc})}}^{+}
\end{aligned}
$$

Following the lines of the derivation of $R$-D Standard ESPRIT with spatial smoothing, $\tilde{\boldsymbol{a}}^{(\mathrm{nc})(r)^{\mathrm{T}}}$ can be simplified as

$$
\tilde{\boldsymbol{a}}^{(\mathrm{nc})(r)^{\mathrm{T}}}=\frac{\sqrt{2 M_{\mathrm{sub}}} M_{\mathrm{sub}_{r}}}{2 M_{\mathrm{sub}}\left(M_{\mathrm{sub}_{r}}-1\right)} \cdot\left[\begin{array}{c}
1 \\
\tilde{\Psi}
\end{array}\right]^{\mathrm{H}} \otimes \check{\boldsymbol{a}}^{(r)^{\mathrm{T}}},
$$

where $\overline{\boldsymbol{a}}^{(r)^{\mathrm{T}}}$ is given in 59). Consequently, the MSE for $R$-D NC Standard ESPRIT with spatial smoothing in (33) can be written as

$$
\mathbb{E}\left\{\left(\Delta \mu^{(r)}\right)^{2}\right\}=\frac{1}{2} \cdot\left(\boldsymbol{z}^{(\mathrm{nc})^{\mathrm{T}}} \boldsymbol{R}_{\mathrm{SS}}^{(\mathrm{nc})} \boldsymbol{z}^{(\mathrm{nc})^{*}}-\operatorname{Re}\left\{\boldsymbol{z}^{(\mathrm{nc})^{\mathrm{T}}} \boldsymbol{C}_{\mathrm{SS}}^{(\mathrm{nc})} \boldsymbol{z}\right\}\right),
$$

where $\boldsymbol{R}_{\mathrm{SS}}^{(\mathrm{nc})}$ and $\boldsymbol{C}_{\mathrm{SS}}^{(\mathrm{nc})}$ are given according to 36 . Next, we use (73) and (75) to express (76) as

$$
\begin{aligned}
\mathbb{E}\left\{\left(\Delta \mu^{(r)}\right)^{2}\right\}=\frac{k^{(\mathrm{nc})^{2}}}{2} \cdot( & \boldsymbol{v}^{(\mathrm{nc})^{\mathrm{T}}} \boldsymbol{R}_{\mathrm{SS}}^{(\mathrm{nc})} \boldsymbol{v}^{(\mathrm{nc})^{*}} \\
- & \left.\operatorname{Re}\left\{\boldsymbol{v}^{(\mathrm{nc})^{\mathrm{T}}} \boldsymbol{C}_{\mathrm{SS}}^{(\mathrm{nc})} \boldsymbol{v}^{(\mathrm{nc})}\right\}\right),
\end{aligned}
$$

where again $\boldsymbol{z}^{(\mathrm{nc})^{\mathrm{T}}}=k^{(\mathrm{nc})} \cdot \boldsymbol{v}^{(\mathrm{nc})^{\mathrm{T}}}$ with $\boldsymbol{v}^{(\mathrm{nc})^{\mathrm{T}}}=\boldsymbol{a}_{L}^{\mathrm{H}} \otimes \overline{\boldsymbol{s}}^{\mathrm{H}} \otimes$ $\left[\begin{array}{c}1 \\ \tilde{\Psi}\end{array}\right]^{\mathrm{H}} \otimes \check{\boldsymbol{a}}^{(r)^{\mathrm{T}}}$ and $k^{(\mathrm{nc})}=\frac{1}{2 N L \hat{P}_{\mathrm{s}}} \cdot \frac{M_{\mathrm{sub}_{r}}}{M_{\mathrm{sub}}\left(M_{\mathrm{sub}_{r}}-1\right)}$. Considering the first term of (77) and expanding $\boldsymbol{R}_{\mathrm{SS}}^{(\mathrm{nc})}$, we apply the same steps as in 61) and 63. As a result, the first term reduces to $4 \cdot \sigma_{\mathrm{n}}^{2} \cdot N \hat{P}_{\mathrm{s}} \cdot \prod_{\substack{p=1 \\ p \neq r}}^{R} c_{p} \cdot \min \left\{L_{r}, M_{r}-L_{r}\right\}$. The second term of (77) can be computed accordingly to obtain $-4 \cdot \sigma_{\mathrm{n}}^{2} \cdot N \hat{P}_{\mathrm{s}}$. $\prod_{p=1}^{R} c_{p} \cdot \min \left\{L_{r}, M_{r}-L_{r}\right\}$.

Using these expressions in (77), the MSE of $R$-D NC Standard ESPRIT with spatial smoothing is given by

$$
\mathbb{E}\left\{\left(\Delta \mu^{(r)}\right)^{2}\right\}=\frac{\sigma_{\mathrm{n}}^{2}}{N \hat{P}_{\mathrm{s}}} \cdot \frac{\min \left\{L_{r}, M_{r}-L_{r}\right\}}{\left(M_{r}-L_{r}\right)^{2} L_{r}^{2}} \cdot \prod_{\substack{p=1 \\ p \neq r}}^{R} \frac{c_{p}}{M_{\mathrm{sub}_{p}}^{2} L_{p}^{2}} .
$$

As this result is equal to 64 and (71), the theorem has been proven.

\section{APPENDIX C \\ Proof of EQUATION (42)}

For the proof, we consider the case $L_{r} \leq \frac{M_{r}}{2}$, however, the derivation for $L_{r}>\frac{M_{r}}{2}$ follows the same steps. The MSE in (39) is given by

$$
\operatorname{MSE}_{\mathrm{SS}}^{(r)} \approx \frac{1}{\hat{\rho}} \cdot \frac{a}{\left(M_{r}-L_{r}\right)^{2} L_{r}} \quad \text { for } \quad L_{r} \leq \frac{M_{r}}{2},
$$

where we have defined $a=\prod_{\substack{p=1 \\ p \neq r}}^{R} \frac{c_{p}}{M_{\operatorname{sub}_{p}}^{2} L_{p}^{2}}$. In order to determine the optimal number of subarrays $L_{r}$ in the $r$-th mode, we minimize (39) with respect to $L_{r}$. That is, we first compute the derivative of 39 with respect to $L_{r}$ given by

$$
\frac{\partial \mathrm{MSE}_{\mathrm{SS}}^{(r)}}{\partial L_{r}}=\frac{1}{\hat{\rho}} \cdot \frac{a\left(M_{r}-3 L_{r}\right)}{\left(L_{r}-M_{r}\right)^{3} L_{r}^{2}}
$$

and then equate 80 to zero and solve for $L_{r}$, yielding

$$
L_{r}^{\mathrm{opt}}=\frac{1}{3} \cdot M_{r},
$$

which is the desired result in 42.

\section{REFERENCES}

[1] J. Steinwandt, F. Roemer, and M. Haardt, "Asymptotic performance analysis of ESPRIT-type algorithms for circular and strictly non-circular sources with spatial smoothing," in Proc. IEEE Int. Conf. on Acoustics, Speech, and Signal Processing (ICASSP), Florence, Italy, May 2014.

[2] D. Nion and N. D. Sidiropoulos, "Tensor algebra and multidimensional harmonic retrieval in signal processing for mimo radar," IEEE Transactions on Signal Processing, vol. 58, no. 11, pp. 5693-5705, Nov. 2010.

[3] H. Cox, "Fundamentals of bistatic active sonar," in Proc. NATO Advanced Study Inst. Underwater Acoustic Data Processing. Norwood, MA: Kluwer, 1989, Y. T. Chan, Ed.

[4] M. Haardt, R. S. Thomä, and A. Richter, "Multidimensional highresolution parameter estimation with applications to channel sounding," in High-Resolution and Robust Signal Processing, pp. 255-338. New York: Marcel Dekker, 2004, Y. Hua, A. Gershman, and Q. Chen, Eds.

[5] X. Liu, N. D. Sidiropoulos, and T. Jiang, "Multidimensional harmonic retrieval with applications in mimo wireless channel sounding," in Space-Time Processing for MIMO Communications, pp. 41-75. New York: Wiley, 2005, A. Gershman and N. Sidiropoulos, Eds.

[6] M. Pesavento, C. F. Mecklenbräuker, and J. F. Böhme, "Multidimensional rank reduction estimator for parametric MIMO channel models," EURASIP Journal on Applied Signal Processing, vol. 2004, no. 9, pp. 1354-1363, Sept. 2004.

[7] M. Haardt and J. A. Nossek, "Simultaneous Schur decomposition of several non-symmetric matrices to achieve automatic pairing in multidimensional harmonic retrieval problems," IEEE Transactions on Signal Processing, vol. 46, no. 1, pp. 161-169, Jan. 1998.

[8] B. D. Rao and K. V. S. Hari, "Performance analysis of ESPRIT and TAM in determining the direction of arrival of plane waves in noise," IEEE Transactions on Acoustics, Speech, and Signal Processing, vol. 37, no. 12, pp. 1990-1995, Dec. 1989.

[9] F. Li, H. Liu, and R. J. Vaccaro, "Performance analysis for DOA estimation algorithms: Unification, simplifications, and observations," IEEE Transactions on Aerospace and Electronic Systems, vol. 29, no. 4, pp. 1170-1184, Oct. 1993.

[10] F. Roemer and M. Haardt, "A framework for the analytical performance assessment of matrix and tensor-based ESPRIT-type algorithms," Sept. 2012, arXiv: 1209.3253 .

[11] F. Roemer, M. Haardt, and G. Del Galdo, "Analytical performance assessment of multi-dimensional matrix- and tensor-based ESPRIT-type algorithms," IEEE Transactions on Signal Processing, vol. 62, pp. 2611 - 2625, May 2014.

[12] P. J. Schreier and L. L. Scharf, Statistical Signal Processing of Complex-Valued Data: The Theory of Improper and Noncircular Signals, Cambridge University Press, 2010.

[13] H. Abeida and J. P. Delmas, "MUSIC-like estimation of direction of arrival for noncircular sources," IEEE Transactions on Signal Processing, vol. 54, no. 7, pp. 2678-2690, July 2006.

[14] A. Ferreol and P. Chevalier, "Higher order direction finding for arbitrary noncircular sources: The NC-2q-MUSIC algorithm," in Proc. European Signal Processing Conference (EUSIPCO), Aalborg, Denmark, Aug. 2010.

[15] A. Ferreol and P. Chevalier, "New insights into second and fourth-order direction finding for non-circular sources," in Proc. IEEE Sensor Array and Multichannel Signal Processing Workshop (SAM), A Coruna, Spain, 2014.

[16] P. Chargé, Y. Wang, and J. Saillard, "A non-circular sources direction finding method using polynomial rooting," Signal Processing, vol. 81, no. 8, pp. 1765-1770, Aug. 2001.

[17] A. Zoubir, P. Chargé, and Y. Wang, "Non circular sources localization with ESPRIT," in Proc. European Conference on Wireless Technology (ECWT), Munich, Germany, Oct. 2003. 
[18] M. Haardt and F. Roemer, "Enhancements of Unitary ESPRIT for noncircular sources," in Proc. IEEE Int. Conf. on Acoust., Speech, and Signal Processing (ICASSP), Montreal, Canada, May 2004.

[19] J. Steinwandt, F. Roemer, M. Haardt, and G. Del Galdo, "R-dimensional ESPRIT-type algorithms for strictly second-order non-circular sources and their performance analysis," IEEE Transactions on Signal Processing, vol. 62, no. 18, pp. 4824-4838, Sept. 2014.

[20] H. Abeida and J. P. Delmas, "Statistical performance of MUSIC-like algorithms in resolving noncircular sources," IEEE Transactions on Signal Processing, vol. 56, no. 9, pp. 4317-4329, Sept. 2008.

[21] F. Gao, A. Nallanathan, and Y. Wang, "mproved music under the coexistence of both circular and noncircular sources," IEEE Transactions on Signal Processing, vol. 56, no. 7, pp. 3033-3038, 2008

[22] J. Steinwandt, F. Roemer, and M. Haardt, "ESPRIT-type algorithms for a received mixture of circular and strictly non-circular signals," in Proc. IEEE Int. Conference on Acoustics, Speech, and Signal Processing (ICASSP), Brisbane, Australia, Apr. 2015.

[23] J. Steinwandt, F. Roemer, and M. Haardt, "Analytical performance assessment of ESPRIT-type algorithms for coexisting circular and strictly non-circular signals," in Proc. IEEE Int. Conference on Acoustics, Speech, and Signal Processing (ICASSP), Shanghai, China, Mar. 2016.

[24] M. Haardt, Efficient One-, Two-, and Multidimensional High-Resolution Array Signal Processing, Technische Universität München, Shaker Verlag, Aachen, 1997.

[25] D. Tse and P. Viswanath, Fundamentals of wireless communications, Cambridge University Press, 2005.

[26] P. Pal and P. P. Vaidyanathan, "Nested arrays: A novel approach to array processing with enhanced degrees of freedom," IEEE Transactions on Signal Processing, vol. 58, no. 8, pp. 4167-4181, Aug. 2010.

[27] P. Comon and G. H. Golub, "Tracking a few extreme singular values and vectors in signal processing," Proceedings of the IEEE, vol. 78, no. 8, pp. 1327-1343, Sept. 1990.

[28] J. E. Evans, J. R. Johnson, and D. F. Sun, "Application of advanced signal processing techniques to angle of arrival estimation in atc navigation and surveillance systems," MIT Lincoln Lab., Tech. Rep., 1982.

[29] T.-J. Shan, M. Wax, and T. Kailath, "On spatial smoothing for directionof-arrival estimation of coherent signals," IEEE Trans. Acoust., Speech, Signal Processing, vol. 33, no. 4, pp. 806-811, Aug. 1985.
[30] S. U. Pillai and B. H. Kwon, "Forward/backward spatial smoothing techniques for coherent signal identification," IEEE Trans. Acoust., Speech, Signal Processing, vol. 37, no. 1, pp. 8-15, Jan. 1989.

[31] S. U. Pillai and B. H. Kwon, "Performance analysis of MUSIC-type high resolution estimators for direction finding in correlated and coherent scenes," IEEE Trans. Acoust., Speech, Signal Processing, vol. 37, no. 8, pp. 1176-1189, Aug. 1989

[32] B. D. Rao and K. V. S. Hari, "Effect of spatial smoothing on the performance of MUSIC and minimum-norm method," Proc. Inst. Elect. Eng. F, vol. 137, pp. 449-458, Dec. 1990.

[33] B. D. Rao and K. V. S. Hari, "Weighted subspace methods and spatial smoothing: Analysis and comparison," IEEE Transactions on Signal Processing, vol. 41, no. 2, pp. 788-803, Feb. 1993.

[34] Y. Hua and T. K. Sarkar, "Matrix pencil method for estimating parameters of exponentially damped/undamped sinusoids in noise," IEEE Transactions on Signal Processing, vol. 38, no. 5, pp. 814-824, May 1990.

[35] K. V. S. Hari and B. V. Ramakrishnan, "Performance analysis of modified spatial smoothing technique for direction estimation," Signal Processing, vol. 79, pp. 73-85, Nov. 1999.

[36] A. N. Lemma, A.-J. van der Veen, and E. F. Deprettere, "Analysis of joint angle-frequency estimation using ESPRIT," IEEE Transactions on Signal Processing, vol. 51, no. 5, pp. 1264-1283, May 2003.

[37] A. J. Weiss and B. Friedlander, "Performance analysis of spatial smoothing with interpolated data," IEEE Transactions on Signal Processing, vol. 41, no. 5, pp. 1881-1892, May 1993.

[38] J. Steinwandt, F. Roemer, M. Haardt, and G. Del Galdo, "Deterministic Cramér-Rao bound for strictly non-circular sources and analytical analysis of the achievable gains," IEEE Transactions on Signal Processing, vol. 64, no. 17, pp. 4417-4431, Sept. 2016.

[39] T. Fu and X. Gao, "Simultaneous diagonalization with similarity transformation for non-defective matrices," in Proc. IEEE Int. Conf. on Acoust., Speech, and Signal Processing (ICASSP), Toulouse, France, May 2006.

[40] J. R. Magnus and H. Neudecker, Matrix differential calculus with applications in statistics and econometrics, John Wiley and Sons, 1995. 